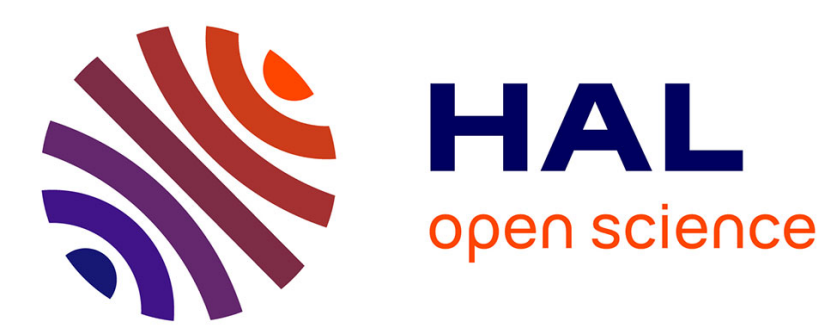

\title{
ASYMPTOTICS OF SYMMETRIC COMPOUND POISSON POPULATION MODELS
}

\author{
Thierry Huillet, Martin Moehle
}

\section{To cite this version:}

Thierry Huillet, Martin Moehle. ASYMPTOTICS OF SYMMETRIC COMPOUND POISSON POPULATION MODELS. Combinatorics, Probability and Computing, 2015, 24 (Special Issue 01), pp.216253. 10.1017/S0963548314000431 . hal-00730734

\section{HAL Id: hal-00730734 https://hal.science/hal-00730734}

Submitted on 11 Sep 2012

HAL is a multi-disciplinary open access archive for the deposit and dissemination of scientific research documents, whether they are published or not. The documents may come from teaching and research institutions in France or abroad, or from public or private research centers.
L'archive ouverte pluridisciplinaire HAL, est destinée au dépôt et à la diffusion de documents scientifiques de niveau recherche, publiés ou non, émanant des établissements d'enseignement et de recherche français ou étrangers, des laboratoires publics ou privés. 


\title{
ASYMPTOTICS OF SYMMETRIC COMPOUND POIS- SON POPULATION MODELS
}

\author{
ThierRy Huillet 1 and Martin MÖHLE 2
}

September 10, 2012

\begin{abstract}
Compound Poisson population models are particular conditional branching process models. A formula for the transition probabilities of the backward process for general compound Poisson models is verified. Symmetric compound Poisson models are defined in terms of a parameter $\theta \in(0, \infty)$ and a power series $\phi$ with positive radius $r$ of convergence. It is shown that the asymptotic behavior of symmetric compound Poisson models is mainly determined by the characteristic value $\theta r \phi^{\prime}(r-)$. If $\theta r \phi^{\prime}(r-) \geq 1$, then the model is in the domain of attraction of the Kingman coalescent. If $\theta r \phi^{\prime}(r-)<1$, then under mild regularity conditions a condensation phenomenon occurs which forces the model to be in the domain of attraction of a discrete-time $\Lambda$-coalescent. The proofs are partly based on the analytic saddle point method. They draw heavily from local limit theorems and from results of S. Janson on simply generated trees, conditioned Galton-Watson trees, random allocations and condensation. Several examples of compound Poisson models are provided and analyzed.
\end{abstract}

Keywords: Bell polynomials; coalescent with multiple collisions; compound Poisson model; condensation; Dirichlet model; Kingman coalescent; saddle point method; Wright-Fisher model

2010 Mathematics Subject Classification: Primary 60F05, 60G09, 60J10, 60J80; Secondary $60 \mathrm{~K} 35,92 \mathrm{D} 10,92 \mathrm{D} 25$

\section{Introduction}

Conditional branching process models, introduced by Karlin and McGregor 14, 15, are population models with fixed population size $N \in \mathbb{N}:=\{1,2, \ldots\}$ and non-overlapping generations. They are defined in terms of a sequence $\left(\xi_{n}\right)_{n \in \mathbb{N}}$ of independent non-negative integer-valued random variables satisfying $\mathbb{P}\left(\xi_{1}+\cdots+\xi_{N}=N\right)>0$. If, for $i \in\{1, \ldots, N\}, \mu_{N, i}$ denotes the number of offspring of the $i$ th individual alive in some fixed generation, then the random variables $\mu_{N, 1}, \ldots, \mu_{N, N}$ have (by definition) joint distribution

$$
\mathbb{P}\left(\mu_{N, 1}=j_{1}, \ldots, \mu_{N, N}=j_{N}\right)=\frac{\mathbb{P}\left(\xi_{1}=j_{1}\right) \cdots \mathbb{P}\left(\xi_{N}=j_{N}\right)}{\mathbb{P}\left(\xi_{1}+\cdots+\xi_{N}=N\right)},
$$

$j_{1}, \ldots, j_{N} \in \mathbb{N}_{0}:=\{0,1, \ldots\}$ with $j_{1}+\cdots+j_{N}=N$. For convenience we will often drop the index $N$ and simply write $\mu_{i}$ instead of $\mu_{N, i}$. For some more information on conditional branching process models we refer the reader to [9, Section 3] and [10.

We now turn to the definition of compound Poisson population models. Let $\theta_{1}, \theta_{2}, \ldots$ be strictly positive real numbers and let $\phi(z)=\sum_{m=1}^{\infty} \phi_{m} z^{m} / m !,|z|<r$, be a power series with radius $r \in(0, \infty]$ of convergence and with non-negative coefficients $\phi_{m} \geq 0, m \in \mathbb{N}$. It is also assumed

\footnotetext{
${ }^{1}$ Laboratoire de Physique Théorique et Modélisation, CNRS-UMR 8089 et Université de Cergy-Pontoise, 2 Avenue Adolphe Chauvin, 95302 Cergy-Pontoise, France, E-mail: thierry.huillet@u-cergy.fr

${ }^{2}$ Mathematisches Institut, Eberhard Karls Universität Tübingen, Auf der Morgenstelle 10, 72076 Tübingen, Germany, E-mail: martin.moehle@uni-tuebingen.de
} 
that $\phi_{1}>0$. Compound Poisson models are particular conditional branching process models where each random variable $\xi_{n}$ has probability generating function (pgf)

$$
f_{n}(x):=\mathbb{E}\left(x^{\xi_{n}}\right)=\exp \left(-\theta_{n}(\phi(z)-\phi(z x))\right), \quad|x| \leq 1 .
$$

In (1), $z$ is viewed as a fixed parameter satisfying $0<z<r$. If $M_{n}$ is a random variable having a Poisson distribution with parameter $\theta_{n} \phi(z)$ and if $X_{1}, X_{2}, \ldots$ are independent random variables and independent of $M_{n}$ each with $\operatorname{pgf} x \mapsto \phi(z x) / \phi(z),|x| \leq 1$, then $\sum_{j=1}^{M_{n}} X_{j}$ has pgf 11 . This subclass of conditional branching process models is therefore called the compound Poisson class. In order to analyze compound Poisson models it is useful to view $z$ as a variable and to introduce, for $\theta \in[0, \infty)$, the Taylor expansion $\exp (\theta \phi(z))=\sum_{k=0}^{\infty} \sigma_{k}(\theta) z^{k} / k !,|z|<r$. The coefficients $\sigma_{k}(\theta)$ depend on $\left(\phi_{m}\right)_{m \in \mathbb{N}}$ and they satisfy the recursion

$$
\sigma_{0}(\theta)=1 \quad \text { and } \quad \sigma_{k+1}(\theta)=\theta \sum_{l=0}^{k}\left(\begin{array}{l}
k \\
l
\end{array}\right) \phi_{k-l+1} \sigma_{l}(\theta), \quad k \in \mathbb{N}_{0}, \theta \in[0, \infty) .
$$

The coefficients $\sigma_{k}(\theta)$ are mainly introduced, since, by (1), the distribution of $\xi_{n}, n \in \mathbb{N}$, satisfies

$$
\mathbb{P}\left(\xi_{n}=k\right)=\frac{\sigma_{k}\left(\theta_{n}\right)}{k !} z^{k} \exp \left(-\theta_{n} \phi(z)\right), \quad k \in \mathbb{N}_{0} .
$$

Note furthermore that $\xi_{1}+\cdots+\xi_{n}, n \in \mathbb{N}$, has distribution

$$
\mathbb{P}\left(\xi_{1}+\cdots+\xi_{n}=k\right)=\frac{\sigma_{k}\left(\Theta_{n}\right)}{k !} z^{k} \exp \left(-\Theta_{n} \phi(z)\right), \quad k \in \mathbb{N}_{0},
$$

where $\Theta_{n}:=\theta_{1}+\cdots+\theta_{n}$. From (2) it follows by induction on $k \in \mathbb{N}$ that $\phi_{k}=\lim _{\theta \rightarrow 0} \sigma_{k}(\theta) / \theta$ for all $k \in \mathbb{N}$, so the coefficients $\phi_{k}, k \in \mathbb{N}$, of the power series $\phi$ can be recovered from the coefficients $\sigma_{k}(\theta), k \in \mathbb{N}_{0}, \theta \in(0, \infty)$. From $\phi_{1}>0$ it follows that $\sigma_{k}(\theta)$ is a polynomial in $\theta$ of degree $k$. In the literature (see, for example, [1] or [4) the $\sigma_{k}(\theta)$ are called the exponential or Bell polynomials. We have $\sigma_{1}(\theta)=\theta \phi_{1}, \sigma_{2}(\theta)=\theta \phi_{2}+\theta^{2} \phi_{1}^{2}, \sigma_{3}(\theta)=\theta \phi_{3}+3 \theta^{2} \phi_{1} \phi_{2}+\theta^{3} \phi_{1}^{3}$, $\sigma_{4}(\theta)=\theta \phi_{4}+\theta^{2}\left(4 \phi_{1} \phi_{3}+3 \phi_{2}^{2}\right)+6 \theta^{3} \phi_{1}^{2} \phi_{2}+\theta^{4} \phi_{1}^{4}$, and so on. The coefficients $B_{k l}\left(\phi_{1}, \phi_{2}, \ldots\right), k \in \mathbb{N}_{0}$, $l \in\{0, \ldots, k\}$, of the polynomials $\sigma_{k}(\theta)=\sum_{l=0}^{k} B_{k l}\left(\phi_{1}, \phi_{2}, \ldots\right) \theta^{l}, k \in \mathbb{N}_{0}$, are called the Bell coefficients.

In the following, for $x \in \mathbb{R}$ and $k \in \mathbb{N}_{0}$, the notation $(x)_{k}:=x(x-1) \cdots(x-k+1)$ and $[x]_{k}:=$ $x(x+1) \cdots(x+k-1)$ is used for the descending and ascending factorials respectively, with the convention that $(x)_{0}:=1$ and $[x]_{0}:=1$. It is readily checked that $\xi_{n}$ has descending factorial moments

$$
\mathbb{E}\left(\left(\xi_{n}\right)_{k}\right)=f_{n}^{(k)}(1)=z^{k} \sum_{l=0}^{k} B_{k l}\left(\phi^{\prime}(z), \phi^{\prime \prime}(z), \ldots\right) \theta_{n}^{l}, \quad n \in \mathbb{N}, k \in \mathbb{N}_{0},
$$

i.e. $\mathbb{E}\left(\xi_{n}\right)=\theta_{n} z \phi^{\prime}(z), \mathbb{E}\left(\left(\xi_{n}\right)_{2}\right)=\theta_{n} z^{2} \phi^{\prime \prime}(z)+\theta_{n}^{2} z^{2}\left(\phi^{\prime}(z)\right)^{2}$ and so on. The descending factorial moments therefore satisfy the recursion

$$
\mathbb{E}\left(\left(\xi_{n}\right)_{k+1}\right)=\theta_{n} \sum_{l=0}^{k}\left(\begin{array}{l}
k \\
l
\end{array}\right) z^{k-l+1} \phi^{(k-l+1)}(z) \mathbb{E}\left(\left(\xi_{n}\right)_{l}\right), \quad n \in \mathbb{N}, k \in \mathbb{N}_{0} .
$$


It is known (see, for example, [9, p. 535]) that $\mu=\left(\mu_{1}, \ldots, \mu_{N}\right)$ has distribution

$$
\mathbb{P}(\mu=j)=\frac{N !}{\sigma_{N}\left(\Theta_{N}\right)} \prod_{n=1}^{N} \frac{\sigma_{j_{n}}\left(\theta_{n}\right)}{j_{n} !}, \quad j=\left(j_{1}, \ldots, j_{N}\right) \in \Delta(N),
$$

where $\Theta_{N}:=\theta_{1}+\cdots+\theta_{N}$ and $\Delta(N)$ denotes the discrete $N$-simplex consisting of all $j=$ $\left(j_{1}, \ldots, j_{N}\right) \in \mathbb{N}_{0}^{N}$ satisfying $j_{1}+\cdots+j_{N}=N$. Note that the distribution of $\mu$ is not necessarily exchangeable. Moreover (see, for example, [10, Eq. (4)]), $\mu$ has joint factorial moments

$$
\mathbb{E}\left(\left(\mu_{1}\right)_{k_{1}} \cdots\left(\mu_{N}\right)_{k_{N}}\right)=\frac{N !}{\sigma_{N}\left(\Theta_{N}\right)} \sum_{\substack{j_{1} \geq k_{1}, \ldots, j_{N} \geq k_{N} \\ j_{1}+\cdots+j_{N}=N}} \frac{\sigma_{j_{1}}\left(\theta_{1}\right) \cdots \sigma_{j_{N}}\left(\theta_{N}\right)}{\left(j_{1}-k_{1}\right) ! \cdots\left(j_{N}-k_{N}\right) !},
$$

$N \in \mathbb{N}, k_{1}, \ldots, k_{N} \in \mathbb{N}_{0}, \theta_{1}, \ldots, \theta_{N} \in(0, \infty)$. For some compound Poisson models, namely for skewed Wright-Fisher models and for skewed Dirichlet models, the alternative and simpler formula (see, for example, [10, Lemma 2.1] and the remarks thereafter)

$$
\mathbb{E}\left(\left(\mu_{1}\right)_{k_{1}} \cdots\left(\mu_{N}\right)_{k_{N}}\right)=(N)_{k} \frac{\sigma_{k_{1}}\left(\theta_{1}\right) \cdots \sigma_{k_{N}}\left(\theta_{N}\right)}{\sigma_{k}\left(\theta_{1}+\cdots+\theta_{N}\right)}
$$

holds for all $k_{1}, \ldots, k_{N} \in \mathbb{N}_{0}$ and all $\theta_{1}, \ldots, \theta_{N} \in(0, \infty)$, where $k:=k_{1}+\cdots+k_{N}$. For some more information on compound Poisson models we refer the reader to [17.

The article is organized as follows. The main results are presented in the following Section 2 . Proofs are provided in Section 3 . Examples of compound Poisson models are studied in Section 4. One purpose of this article is to provide proofs of the results stated without proof in [10].

\section{Results}

The following Proposition 2.1. stated without proof in 10, Proposition 2.2], provides expressions for the transition probabilities $\widehat{P}_{i, j}$ of the backward process $\widehat{X}$ for an arbitrary compound Poisson model. The proof of Proposition 2.1 is provided in Section 3 .

Proposition 2.1 If, for each $n \in \mathbb{N}$, the random variable $\xi_{n}$ has a pgf of the form (1), then the backward process $\widehat{X}$ of the associated compound Poisson model has transition probabilities

$$
\begin{aligned}
\widehat{P}_{i, j}= & \frac{1}{\left(\begin{array}{c}
N \\
i
\end{array}\right)} \sum_{1 \leq n_{1}<\cdots<n_{j} \leq N} \sum_{\substack{k_{1}, \ldots, k_{j} \in \mathbb{N}_{0} \\
k:=k_{1}+\cdots+k_{j} \leq N}} \frac{(N)_{k}}{k_{1} ! \cdots k_{j} !} . \\
& \frac{\left(\prod_{i=1}^{j} \sigma_{k_{i}}\left(\theta_{n_{i}}\right)\right) \sigma_{N-k}\left(\Theta_{N}-\sum_{i=1}^{j} \theta_{n_{i}}\right)}{\sigma_{N}\left(\Theta_{N}\right)} \sum_{\substack{l_{1}, \ldots, l_{j} \in \mathbb{N} \\
l_{1}+\cdots+l_{j}=i}}\left(\begin{array}{c}
k_{1} \\
l_{1}
\end{array}\right) \cdots\left(\begin{array}{c}
k_{j} \\
l_{j}
\end{array}\right), \quad i, j \in S,
\end{aligned}
$$

with the convention that $\widehat{P}_{i, 0}=\delta_{i 0}, i \in S$. Here $\Theta_{N}:=\theta_{1}+\cdots+\theta_{N}, S:=\{0, \ldots, N\}$, and the coefficients $\sigma_{k}(\theta), k \in \mathbb{N}_{0}, \theta \in[0, \infty)$, are recursively defined via (2). In particular,

$$
\widehat{P}_{i, 1}=\sum_{n=1}^{N} \sum_{k=i}^{N}\left(\begin{array}{c}
N-i \\
k-i
\end{array}\right) \frac{\sigma_{k}\left(\theta_{n}\right) \sigma_{N-k}\left(\Theta_{N}-\theta_{n}\right)}{\sigma_{N}\left(\Theta_{N}\right)}, \quad i \in\{1, \ldots, N\} .
$$


Remark. Proposition 2.1 in particular yields the coalescence probability

$$
c_{N}:=\widehat{P}_{2,1}=\sum_{n=1}^{N} \sum_{k=2}^{N}\left(\begin{array}{c}
N-2 \\
k-2
\end{array}\right) \frac{\sigma_{k}\left(\theta_{n}\right) \sigma_{N-k}\left(\Theta_{N}-\theta_{n}\right)}{\sigma_{N}\left(\Theta_{N}\right)}, \quad N \geq 2,
$$

and the probability

$$
d_{N}:=\widehat{P}_{3,1}=\sum_{n=1}^{N} \sum_{k=3}^{N}\left(\begin{array}{c}
N-3 \\
k-3
\end{array}\right) \frac{\sigma_{k}\left(\theta_{n}\right) \sigma_{N-k}\left(\Theta_{N}-\theta_{n}\right)}{\sigma_{N}\left(\Theta_{N}\right)}, \quad N \geq 3,
$$

that three individuals, picked at random and without replacement from some arbitrary but fixed generation of the population, share a common parent.

In the following we focus on the unbiased (symmetric) case, when all the parameters $\theta_{n}=\theta$ are equal to some constant $\theta \in(0, \infty)$. In this case the distribution (4) of $\mu$ is exchangeable and (7) reduces to

$$
\widehat{P}_{i, j}=\frac{\left(\begin{array}{c}
N \\
j
\end{array}\right)}{\left(\begin{array}{c}
N \\
i
\end{array}\right)} \sum_{\substack{k_{1}, \ldots, k_{j} \in \mathbb{N}_{0} \\
k:=k_{1}+\cdots+k_{j} \leq N}} \frac{(N)_{k}}{k_{1} ! \cdots k_{j} !} \frac{\left(\prod_{i=1}^{j} \sigma_{k_{i}}(\theta)\right) \sigma_{N-k}((N-j) \theta)}{\sigma_{N}(N \theta)} \sum_{\substack{l_{1}, \ldots, l_{j} \in \mathbb{N} \\
l_{1}+\cdots+l_{j}=i}}\left(\begin{array}{c}
k_{1} \\
l_{1}
\end{array}\right) \cdots\left(\begin{array}{c}
k_{j} \\
l_{j}
\end{array}\right),
$$

$i, j \in S$, with the convention that $\widehat{P}_{i, 0}=\delta_{i 0}, i \in S$. In particular,

$$
\begin{gathered}
\widehat{P}_{i, 1}=N \sum_{k=i}^{N}\left(\begin{array}{c}
N-i \\
k-i
\end{array}\right) \frac{\sigma_{k}(\theta) \sigma_{N-k}((N-1) \theta)}{\sigma_{N}(N \theta)}, \quad i \in\{1, \ldots, N\}, \\
c_{N}=N \sum_{k=2}^{N}\left(\begin{array}{c}
N-2 \\
k-2
\end{array}\right) \frac{\sigma_{k}(\theta) \sigma_{N-k}((N-1) \theta)}{\sigma_{N}(N \theta)}, \quad N \geq 2,
\end{gathered}
$$

and

$$
d_{N}=N \sum_{k=3}^{N}\left(\begin{array}{c}
N-3 \\
k-3
\end{array}\right) \frac{\sigma_{k}(\theta) \sigma_{N-k}((N-1) \theta)}{\sigma_{N}(N \theta)}, \quad N \geq 3,
$$

in agreement with (8), (9), and (10).

We are interested in the asymptotic behavior of symmetric compound Poisson models as $N \rightarrow \infty$. The following results show that the characteristic value $\operatorname{\theta r} \phi^{\prime}(r-) \in(0, \infty]$ is of fundamental interest in this context, where $r \in(0, \infty]$ denotes the radius of convergence of $\phi$. Theorem 2.2 below, stated in slightly different form and without proof in 10, Theorem 2.3], clarifies that many symmetric compound Poisson models are in the domain of attraction of the Kingman coalescent.

Theorem 2.2 (supercritical case) Suppose that $\theta r \phi^{\prime}(r-) \in(1, \infty]$ such that the equation $\theta z \phi^{\prime}(z)=1$ has a unique real solution $z(\theta) \in(0, r)$. Then, $\mu_{N, 1} \rightarrow X$ in distribution as $N \rightarrow \infty$ with convergence $\mathbb{E}\left(\mu_{N, 1}^{p}\right) \rightarrow \mathbb{E}\left(X^{p}\right)$ as $N \rightarrow \infty, p \in(0, \infty)$, of all moments, where $X$ is a non-negative integer-valued random variable with distribution

$$
\mathbb{P}(X=k)=\frac{\sigma_{k}(\theta)}{k !}(z(\theta))^{k} \exp (-\theta \phi(z(\theta))), \quad k \in \mathbb{N}_{0},
$$


and mean $\mathbb{E}(X)=1$. Moreover, in the sense of [9, Definition 2.1 (a)], the associated symmetric compound Poisson population model is in the domain of attraction of the Kingman coalescent. The effective population size $N_{e}:=1 / c_{N}$ satisfies $N_{e} \sim \varrho N$ as $N \rightarrow \infty$ with $\varrho:=1 / \operatorname{Var}(X)=$ $1 /\left(1+\theta(z(\theta))^{2} \phi^{\prime \prime}(z(\theta))\right) \in(0,1]$.

\section{Remarks.}

1. Two independent proofs of Theorem 2.2 are provided in Section 3 , The first proof involves the saddle point method and the second proof is based on the local limit theorem. Both proofs differ significantly from the erroneous proof in [9]. To the best of the authors knowledge these proofs are the first approaches where saddle point methods or, alternatively, local limit theorems are used to establish convergence to the Kingman coalescent. The distribution of the limiting variable $X$ coincides with the distribution (3) of $\xi_{1}$ with the parameter $z$ in (3) replaced by $z(\theta)$. Note that $X$ has mean $\mathbb{E}(X)=\theta z(\theta) \phi^{\prime}(z(\theta))=1$. Thus, conditioning $\xi_{1}$ on the event that $\xi_{1}+\cdots+\xi_{N}=N$ and afterwards taking $N \rightarrow \infty$, has altogether the effect that the distribution of $\xi_{1}$ is 'nearly' recovered. Only the information about the mean of $\xi_{1}$ is lost.

2. If $\phi^{\prime}(r-)=\infty$, then for all $\theta \in(0, \infty)$ the equation $\theta z \phi^{\prime}(z)=1$ has a solution $z(\theta) \in(0, r)$. If $\phi^{\prime}(r-)<\infty$, then a solution $z(\theta) \in(0, r)$ of the equation $\theta z \phi^{\prime}(z)=1$ exists if and only if $\theta r \phi^{\prime}(r-)>1$. Examples satisfying $\phi^{\prime}(r-)<\infty$ are provided in Section 4 . The solution $z(\theta)$ (if it exists) is unique since the map $z \mapsto z \phi^{\prime}(z)$ is strictly increasing on $(0, r)$. Closed expressions for the solution $z=z(\theta)$ of the equation $u(z):=z \phi^{\prime}(z)=1 / \theta$ seem to be not available in general. By the inversion formula of Lagrange (see, for example, [5, Section 3.8]), for $k \in \mathbb{N}$ and $x \in(0, \infty)$,

$$
\left[x^{k}\right] u^{-1}(x)=\frac{\left[z^{k-1}\right](z / u(z))^{k}}{k}=\frac{\left[z^{k-1}\right]\left(1 / \phi^{\prime}(z)\right)^{k}}{k}=B_{k-1, k}\left(\psi_{0}, \psi_{1}, \ldots\right),
$$

where $\psi_{n}:=\psi^{(n)}(0), n \in \mathbb{N}_{0}$, with $\psi(z):=1 / \phi^{\prime}(z)$. Choosing $x:=1 / \theta$ and noting that $z(\theta)=u^{-1}(x)$ yields the formal expansion

$$
z(\theta)=\sum_{k=1}^{\infty} \frac{\left[z^{k-1}\right](\psi(z))^{k}}{k} \theta^{-k}=\sum_{k=1}^{\infty} B_{k-1, k}\left(\psi_{0}, \psi_{1}, \ldots\right) \theta^{-k}
$$

Note however that, depending on $\phi$ and $\theta$, this series may not converge, so we can only speak about a formal series here. Alternatively one may approximate the root $z(\theta)$ of the map $z \mapsto \theta z \phi^{\prime}(z)-1$ numerically.

Theorem 2.2 is not applicable if $\theta r \phi^{\prime}(r-) \leq 1$. In this case we have $\phi^{\prime}(r-)<\infty$, which implies that $\phi(r-)<\infty$, since $z \phi^{\prime}(z)=\sum_{m=1}^{\infty} \phi_{m} z^{m} /(m-1) ! \geq \sum_{m=1}^{\infty} \phi_{m} z^{m} / m !=\phi(z)$ for all $z \in[0, r)$. Moreover, from $\phi(r-)<\infty$ it follows that $r<\infty$. Assume from now on that $\operatorname{\theta r} \phi^{\prime}(r-) \leq 1$ and introduce a non-negative integer-valued random variable $X$ with distribution

$$
\mathbb{P}(X=k)=\frac{\sigma_{k}(\theta)}{k !} r^{k} \exp (-\theta \phi(r-)), \quad k \in \mathbb{N}_{0}
$$

Note that $X$ has pgf $s \mapsto \mathbb{E}\left(s^{X}\right)=\exp (-\theta(\phi(r-)-\phi((r s)-))),|s| \leq 1$, and characteristic function $t \mapsto \mathbb{E}(\exp (i t X))=\exp \left(-\theta\left(\phi(r-)-\phi\left(\left(r e^{i t}\right)-\right)\right)\right), t \in \mathbb{R}$. In particular, $\mathbb{E}(X)=\theta r \phi^{\prime}(r-) \leq 1$. 
Moreover, $\mathbb{E}\left(X^{k}\right)<\infty$ if and only if $\phi^{(k)}(r-)<\infty, k \in \mathbb{N}$. Our second asymptotic result, Theorem 2.3 below, addresses the critical case $\mathbb{E}(X)=\theta r \phi^{\prime}(r-)=1$. The subcritical case $\mathbb{E}(X)<1$ is considered at the end of this section.

Theorem 2.3 (critical case, convergence) If $\theta r \phi^{\prime}(r-)=1$, then $\lim _{N \rightarrow \infty} c_{N} \rightarrow 0$ and, in the sense of [9, Definition 2.1 (a)], the associated symmetric compound Poisson population model is in the domain of attraction of the Kingman coalescent.

In general $c_{N}$ can tend to zero very slowly. The proof of Theorem 2.3 , provided in Section 3 , does not provide direct information on the speed of convergence. Under additional assumptions more can be said about the asymptotics of $c_{N}$. The proof of the following Theorem 2.4 draws solely but heavily from local limit theorems. For examples we refer the reader to Section 4 from Example 4.6 on.

Theorem 2.4 (critical case, speed of convergence) Assume that $\theta r \phi^{\prime}(r-)=1$.

a) If the random variable $X$ with distribution (16) satisfies $\mathbb{E}\left(X^{2}\right)<\infty$ or, equivalently, if $\phi^{\prime \prime}(r-)<\infty$, then $\mu_{N, 1} \rightarrow X$ in distribution as $N \rightarrow \infty$. Moreover, the coalescence probability $c_{N}$ satisfies $c_{N} \sim \operatorname{Var}(X) / N$ as $N \rightarrow \infty$, where $\operatorname{Var}(X)=1+\theta r^{2} \phi^{\prime \prime}(r-)$.

b) If there exists a constant $c=c(\theta)>0$ such that the distribution $\sqrt{16}$ ) of the random variable $X$ satisfies $\mathbb{P}(X=k) \sim c k^{-3}$ as $k \rightarrow \infty$, then $\mu_{N, 1} \rightarrow X$ in distribution as $N \rightarrow \infty$. Moreover, the coalescence probability $c_{N}$ satisfies

$$
c_{N} \sim \frac{c}{2} \frac{\log N}{N}, \quad N \rightarrow \infty
$$

c) If there exist constants $\alpha \in(1,2)$ and $\kappa=\kappa(\alpha) \in(0, \infty)$ such that the power series $\phi$ of the compound Poisson model satisfies

$$
\phi(z)=\phi(r-)+\phi^{\prime}(r-)(z-r)+\kappa(r-z)^{\alpha}+O\left((r-z)^{2}\right), \quad z \rightarrow r,
$$

then the distribution (16) of the random variable $X$ satisfies $\mathbb{P}(X=k) \sim c k^{-\alpha-1}$ as $k \rightarrow \infty$ with $c:=\theta \kappa r^{\alpha} / \Gamma(-\alpha)$. Moreover, $\mu_{N, 1} \rightarrow X$ in distribution as $N \rightarrow \infty$ and the coalescence probability $c_{N}$ satisfies

$$
c_{N} \sim c \frac{\int_{0}^{\infty} x^{1-\alpha} g(-x) d x}{g(0)} N^{2(1-\alpha) / \alpha}, \quad N \rightarrow \infty .
$$

Here $g$ denotes the density of an $\alpha$-stable random variable with characteristic function $t \mapsto$ $\exp \left(d(-i t)^{\alpha}\right), t \in \mathbb{R}$, where $d:=c \Gamma(-\alpha)=\theta \kappa r^{\alpha}$.

\section{Remarks.}

1. Assume that $\alpha \in(1,2)$. Lemma 5.2, applied with $\beta:=1-\alpha \in(-1,0)$, shows that $\int_{0}^{\infty} x^{1-\alpha} g(-x) d x=d^{(1-\alpha) / \alpha} \Gamma(1-\alpha) / \Gamma((1-\alpha) / \alpha)$ and $g(0)=d^{-1 / \alpha}|\Gamma(-1 / \alpha)|^{-1}$. Thus, the fraction in $(19)$ can be expressed in terms of the gamma function via

$$
\frac{\int_{0}^{\infty} x^{1-\alpha} g(-x) d x}{g(0)}=d^{(2-\alpha) / \alpha} \frac{\Gamma(1-\alpha)|\Gamma(-1 / \alpha)|}{\Gamma((1-\alpha) / \alpha)}, \quad \alpha \in(1,2) .
$$


2. Theorem 2.4 b) can be viewed as the boundary case $\alpha=2$ of Theorem 2.4 c). Note that, under the assumptions of Theorem 2.4 b) or c) respectively,

$$
\frac{\mathbb{E}\left(X^{2} 1_{\{X \leq N\}}\right)}{N}=\frac{1}{N} \sum_{k=1}^{N} k^{2} \mathbb{P}(X=k) \sim \frac{c}{N} \sum_{k=1}^{N} k^{1-\alpha} \sim \begin{cases}\frac{c}{2-\alpha} N^{1-\alpha} & \text { for } \alpha \in(1,2), \\ c \frac{\log N}{N} & \text { for } \alpha=2 .\end{cases}
$$

The naive prospect, based on the equation $(N-1) c_{N}=\mathbb{E}\left(\left(\mu_{N, 1}\right)_{2}\right)$, that the coalescence probability $c_{N}$ could be asymptotically equal to $(20)$, is wrong due to Theorem $\left.2.4 \mathrm{~b}\right)$ and c).

Theorems 2.2, 2.3. and 2.4 are not applicable if $\mathbb{E}(X)<1$. In this subcritical case it seems to be not straightforward to derive asymptotic results without further regularity assumptions on the weights $w_{k}:=\sigma_{k}(\theta) / k !, k \in \mathbb{N}_{0}$. Our last asymptotic result below essentially states that a 'condensation phenomenon' occurs which forces the model to be in the domain of attraction of a discrete-time $\Lambda$-coalescent with $\Lambda(d t) / t^{2}$ being a Dirac measure (Dirac coalescent). The result in particular shows that, under certain circumstances, Dirac coalescents arise naturally in the limit as the total population size $N$ tends to infinity and indicates that Dirac coalescents are more important in the context of ancestral population genetics as it seems to be at a first glance.

Theorem 2.5 (subcritical case) Fix $\theta \in(0, \infty)$ and assume that there exist constants $c>0$ and $\beta>2$ such that $w_{k}:=\sigma_{k}(\theta) / k ! \sim c k^{-\beta} / r^{k}$ as $k \rightarrow \infty$. If $\theta r \phi^{\prime}(r-)<1$, then $\mu_{N, 1} \rightarrow X$ in distribution as $N \rightarrow \infty$, where $X$ is a non-negative integer-valued random variable with distribution (16). Moreover, $\lim _{N \rightarrow \infty} c_{N}=u^{2}$, where $u:=1-\mathbb{E}(X)=1-\theta r \phi^{\prime}(r-) \in(0,1)$ and, in the sense of [9, Definition 2.1 (b)], the associated symmetric compound Poisson population model is in the domain of attraction of a discrete-time $\Lambda$-coalescent with $\Lambda:=u^{2} \delta_{u}$.

\section{Remarks.}

1. Note that $\lim _{N \rightarrow \infty} \widehat{P}_{i, j}=\left(\begin{array}{c}i \\ j-1\end{array}\right) u^{i-j+1}(1-u)^{j-1}$ for all $i, j \in \mathbb{N}$ with $i>j$.

2. The physical image is the one of a very prolific individual giving birth to a fixed fraction $u$ of the total population, the others adjusting their random offspring to ensure a constant population size $N$ in each generation. This 'condensation phenomenon' is reminiscent of the one observed by Eldon and Wakeley [6] (see also [11, Proposition 4]) in the context of extended Moran models. However, as observed in [9, Proposition 3.1], compound Poisson models and extended Moran models constitute disjoint reproduction laws.

3. It remains unclear whether Theorem 2.5 holds without any regularity condition on the weight sequence $\left(w_{k}\right)_{k \in \mathbb{N}_{0}}$. Note that, for arbitrary weights (not necessarily coming from a compound Poisson model), theorems of this form do not hold without any regularity condition on the weight sequence $\left(w_{k}\right)_{k \in \mathbb{N}_{0}}$. We refer the reader to the remark before Example 19.33 and to the Examples 19.37 and 19.38 of 13 for analog comments and more details. In our situation however, the weights are coming from a compound Poisson model and have the particular structure $\omega_{k}=\sigma_{k}(\theta) / k$ !. Thus, our weights cannot be arbitrarily irregular and hence, there is some chance that Theorem 2.5 could hold without any conditions on $\left(w_{k}\right)_{k \in \mathbb{N}_{0}}$. We leave this problem open for future work. 


\section{Proofs}

Proof. (of Proposition 2.1) For $j \in\{1, \ldots, N\}$, pairwise distinct $n_{1}, \ldots, n_{j} \in\{1, \ldots, N\}$, and $k_{1}, \ldots, k_{j} \in \mathbb{N}_{0}$ with $k:=k_{1}+\cdots+k_{j} \leq N$ we have

$$
\begin{aligned}
\mathbb{P}\left(\mu_{n_{1}}=k_{1}, \ldots, \mu_{n_{j}}=k_{j}\right) & =\frac{\left(\prod_{i=1}^{j} \mathbb{P}\left(\xi_{n_{i}}=k_{i}\right)\right) \mathbb{P}\left(\sum_{m \in[N] \backslash\left\{n_{1}, \ldots, n_{j}\right\}} \xi_{m}=N-k\right)}{\mathbb{P}\left(\xi_{1}+\cdots+\xi_{N}=N\right)} \\
& =\frac{(N)_{k}}{k_{1} ! \cdots k_{j} !} \frac{\sigma_{k_{1}}\left(\theta_{n_{1}}\right) \cdots \sigma_{k_{j}}\left(\theta_{n_{j}}\right) \sigma_{N-k}\left(\Theta_{N}-\sum_{i=1}^{j} \theta_{n_{i}}\right)}{\sigma_{N}\left(\Theta_{N}\right)}
\end{aligned}
$$

and, therefore, for $l_{1}, \ldots, l_{j} \in \mathbb{N}_{0}$,

$$
\mathbb{E}\left(\prod_{i=1}^{j}\left(\begin{array}{c}
\mu_{n_{i}} \\
l_{i}
\end{array}\right)\right)=\sum_{k_{1}, \ldots, k_{j}}\left(\prod_{i=1}^{j}\left(\begin{array}{c}
k_{i} \\
l_{i}
\end{array}\right)\right) \frac{(N)_{k}}{k_{1} ! \cdots k_{j} !} \frac{\sigma_{k_{1}}\left(\theta_{n_{1}}\right) \cdots \sigma_{k_{j}}\left(\theta_{n_{j}}\right) \sigma_{N-k}\left(\Theta_{N}-\sum_{i=1}^{j} \theta_{n_{i}}\right)}{\sigma_{N}\left(\Theta_{N}\right)},
$$

where the sum $\sum_{k_{1}, \ldots, k_{j}}$ extends over all $k_{1}, \ldots, k_{j} \in \mathbb{N}_{0}$ satisfying $k:=k_{1}+\cdots+k_{j} \leq N$. Thus (7) follows from [9, Eq. (4)]. For $j=1$, 77 reduces to 8). Alternatively, 8) follows as well via

$$
\begin{aligned}
\widehat{P}_{i, 1} & =\sum_{n=1}^{N} \frac{\mathbb{E}\left(\left(\mu_{n}\right)_{i}\right)}{(N)_{i}}=\sum_{n=1}^{N} \sum_{k=i}^{N} \frac{(k)_{i}}{(N)_{i}} \mathbb{P}\left(\mu_{n}=k\right) \\
& =\sum_{n=1}^{N} \sum_{k=i}^{N} \frac{(k)_{i}}{(N)_{i}}\left(\begin{array}{c}
N \\
k
\end{array}\right) \frac{\sigma_{k}\left(\theta_{n}\right) \sigma_{N-k}\left(\Theta_{N}-\theta_{n}\right)}{\sigma_{N}\left(\Theta_{N}\right)} \\
& =\sum_{n=1}^{N} \sum_{k=i}^{N}\left(\begin{array}{c}
N-i \\
k-i
\end{array}\right) \frac{\sigma_{k}\left(\theta_{n}\right) \sigma_{N-k}\left(\Theta_{N}-\theta_{n}\right)}{\sigma_{N}\left(\Theta_{N}\right)} .
\end{aligned}
$$

Proof. (of Theorem 2.2 Two independent proofs are provided. The first proof exploits the analytic saddle point method. The second proof is based on the standard local limit theorem for sequences of independent and identically distributed random variables with finite and non-vanishing variance. We think that both proofs are worthwhile, since they demonstrate the intertwining of analysis and probability.

Proof 1. Fix $k, l \in \mathbb{N}_{0}$ and $\theta \in(0, \infty)$. Let us verify that

$$
\frac{\sigma_{n-k}((n-l) \theta)}{(n-k) !} \sim \frac{(a(\theta))^{n}}{\sqrt{2 \pi n}} b_{k l}(\theta), \quad n \rightarrow \infty,
$$

where

$$
a(\theta):=\frac{e^{\theta \phi(z(\theta))}}{z(\theta)} \quad \text { and } \quad b_{k l}(\theta):=\frac{(z(\theta))^{k} e^{-l \theta \phi(z(\theta))}}{\sqrt{1+\theta(z(\theta))^{2} \phi^{\prime \prime}(z(\theta))}} .
$$

We proceed similarly as in the proof of [4, Theorem 2.1]. However, note that in [4] asymptotic expansions for $\sigma_{n}(\theta)$ are provided whereas we are essentially interested in the asymptotics of $\sigma_{n}(n \theta)$. By Cauchy's integral formula, $\sigma_{n}(\theta) / n !=(2 \pi i)^{-1} \int_{C} z^{-(n+1)} e^{\theta \phi(z)} d z, n \in \mathbb{N}_{0}$, where $C$ is some contour around the origin. Replacing $n$ by $n-k$ and $\theta$ by $(n-l) \theta$ it follows that

$$
\frac{\sigma_{n-k}((n-l) \theta)}{(n-k) !}=\frac{1}{2 \pi i} \int_{C} \frac{e^{(n-l) \theta \phi(z)}}{z^{n-k+1}} d z=\frac{1}{2 \pi i} \int_{C} h(z) e^{n g(z)} d z,
$$


where $h(z):=z^{k-1} e^{-l \theta \phi(z)}$ and $g(z):=\theta \phi(z)-\log z$. Note that $g^{\prime}(z)=\theta \phi^{\prime}(z)-1 / z$ and that $g^{\prime \prime}(z)=\theta \phi^{\prime \prime}(z)+1 / z^{2}$. In particular, $g^{\prime}$ has a single real zero in the interval $(0, r)$ at the point $z(\theta)$ solving the equation $\theta z(\theta) \phi^{\prime}(z(\theta))=1$. Note that $g^{\prime \prime}(z)>0$ for all $z \in(0, r)$. In order to derive the asymptotics of the integral $\int_{C} h(z) e^{n g(z)} d z$ we use the saddle point method (see, for example, [3] or [7] for general references) and choose the contour $C$ to be the circle around the origin with radius $z(\theta)$ such that it passes through the zero $z(\theta)$ of $g^{\prime}$. Note that $g^{\prime}(z(\theta))=0$, so $g\left(z(\theta) e^{i t}\right)$ has Taylor expansion

$$
\begin{aligned}
g\left(z(\theta) e^{i t}\right) & =\sum_{j=0}^{\infty} \frac{g^{(j)}(z(\theta))}{j !}(z(\theta))^{j}\left(e^{i t}-1\right)^{j} \\
& =g(z(\theta))+\frac{g^{\prime \prime}(z(\theta))}{2}(z(\theta))^{2}\left(e^{i t}-1\right)^{2}+\frac{g^{\prime \prime \prime}(z(\theta))}{3 !}(z(\theta))^{3}\left(e^{i t}-1\right)^{3}+O\left(t^{4}\right)
\end{aligned}
$$

leading to the Taylor expansions $\operatorname{Re}\left(g\left(z(\theta) e^{i t}\right)\right)=g(z(\theta))-(z(\theta))^{2} g^{\prime \prime}(z(\theta)) t^{2} / 2+O\left(t^{4}\right)$ and $\operatorname{Im}\left(g\left(z(\theta) e^{i t}\right)\right)=O\left(t^{3}\right)$. The saddle point method yields the asymptotics

$$
\int_{C} h(z) e^{n g(z)} d z \sim i \sqrt{\frac{2 \pi}{n g^{\prime \prime}(z(\theta))}} e^{n g(z(\theta))} h(z(\theta)) .
$$

Dividing this expression by $2 \pi i$ and writing $z$ instead of $z(\theta)$ for convenience yields

$$
\frac{\sigma_{n-k}((n-l) \theta)}{(n-k) !} \sim \frac{e^{n g(z)} h(z)}{\sqrt{2 \pi n g^{\prime \prime}(z)}}=\frac{1}{\sqrt{2 \pi n}}(a(\theta))^{n} b_{k l}(\theta)
$$

with $a(\theta):=e^{g(z)}=e^{\theta \phi(z)} / z$ and $b_{k l}(\theta):=h(z) / \sqrt{g^{\prime \prime}(z)}=z^{k-1} e^{-l \theta \phi(z)} / \sqrt{z^{-2}+\theta \phi^{\prime \prime}(z)}=$ $z^{k} e^{-l \theta \phi(z)} / \sqrt{1+\theta z^{2} \phi^{\prime \prime}(z)}$. Thus, 21 is established. Note that for $k=l=0$ we have

$$
\frac{\sigma_{n}(n \theta)}{n !}=\frac{1}{2 \pi i} \int_{C} \frac{e^{n g(z)}}{z} d z=\frac{1}{2 \pi i} \int_{-\pi}^{\pi} \frac{e^{n g\left(z(\theta) e^{i t}\right)}}{z(\theta) e^{i t}} i z(\theta) e^{i t} d t=\frac{1}{2 \pi} \int_{-\pi}^{\pi} e^{n g\left(z(\theta) e^{i t}\right)} d t .
$$

Taking the real part yields

$$
\frac{\sigma_{n}(n \theta)}{n !}=\frac{1}{2 \pi} \int_{-\pi}^{\pi} \operatorname{Re}\left(e^{n g\left(z(\theta) e^{i t}\right)}\right) d t \sim \frac{1}{2 \pi} \int_{-\pi}^{\pi} e^{n \operatorname{Re}\left(g\left(z(\theta) e^{i t}\right)\right)} d t,
$$

where the last asymptotics is based on the Laplace method as follows. Choose a sequence $\left(\delta_{n}\right)_{n \in \mathbb{N}}$ of positive real numbers satisfying $n \delta_{n}^{2} \rightarrow \infty$ and $n \delta_{n}^{3} \rightarrow 0$, for example, $\delta_{n}:=n^{-\alpha}$ for some fixed $\alpha \in(1 / 3,1 / 2)$. Decomposing the first integral in $(23)$ into the two parts

$$
I_{1}:=\int_{-\delta_{n}}^{\delta_{n}} \operatorname{Re}\left(e^{n g\left(z(\theta) e^{i t}\right)}\right) d t \quad \text { and } \quad I_{2}:=\int_{\left\{\delta_{n}<|t| \leq \pi\right\}} \operatorname{Re}\left(e^{n g\left(z(\theta) e^{i t}\right)}\right) d t
$$

we can approximate $I_{1}$ and show that $I_{2}$ is negligible (in comparison to $I_{1}$ ) for large $n$. Obviously, $1-x^{2} / 2 \leq \cos x \leq 1$ for all $x \in \mathbb{R}$. Choosing $x:=n \operatorname{Im}\left(g\left(z(\theta) e^{i t}\right)\right)$ and using $\operatorname{Im}\left(g\left(z(\theta) e^{i t}\right)\right)=O\left(t^{3}\right)$ and $n t^{3} \rightarrow 0$ as $n \rightarrow \infty$ uniformly for all $|t| \leq \delta_{n}$ it follows that

$$
\lim _{n \rightarrow \infty} \sup _{|t| \leq \delta_{n}}\left|\cos \left(n \operatorname{Im}\left(g\left(z(\theta) e^{i t}\right)\right)\right)-1\right|=0 .
$$


Thus, as $n \rightarrow \infty$, the map $t \mapsto \cos \left(n \operatorname{Im}\left(g\left(z(\theta) e^{i t}\right)\right)\right)$ converges uniformly on $\left[-\delta_{n}, \delta_{n}\right]$ to the constant map $t \mapsto 1$, which implies that

$$
I_{1}=\int_{-\delta_{n}}^{\delta_{n}} \cos \left(n \operatorname{Im}\left(g\left(z(\theta) e^{i t}\right)\right)\right) e^{n \operatorname{Re}\left(g\left(z(\theta) e^{i t}\right)\right)} d t \sim \int_{-\delta_{n}}^{\delta_{n}} e^{n \operatorname{Re}\left(g\left(z(\theta) e^{i t}\right)\right)} d t .
$$

Let us now turn to the second integral $I_{2}$. Define the two functions $f: \mathbb{R} \rightarrow \mathbb{R}$ and $m: \mathbb{R} \rightarrow \mathbb{R}$ via $f(t):=\operatorname{Re}\left(\phi\left(z(\theta) e^{i t}\right)\right)=\sum_{m=1}^{\infty}\left(\phi_{m} / m !\right)(z(\theta))^{m} \cos (m t)$ and $m(t):=\operatorname{Re}\left(g\left(z(\theta) e^{i t}\right)\right)=$ $\operatorname{Re}\left(\theta \phi\left(z(\theta) e^{i t}\right)-\log \left(z(\theta) e^{i t}\right)\right)=\theta f(t)-\log (z(\theta))$ for all $t \in \mathbb{R}$. Note that $f$ is $2 \pi$-periodic and that $f(t)=f(-t)$ for all $t \in \mathbb{R}$. The same holds for the function $m$ since it is a linear transformation of $f$. For $m \in \mathbb{N}$ define $a_{m}:=\phi_{m}(z(\theta))^{m} / m$ ! and choose some $q \in(1, r / z(\theta))$. Then, $\sum_{m=1}^{\infty} a_{m} q^{m}=\phi(z(\theta) q)<\infty$, since $z(\theta) q<r$. By Lemma 5.1 (applied for the function $f$ and with $\varepsilon:=\delta_{n}$ ) it follows that there exists a constant $n_{0} \in \mathbb{N}$ (which may depend on $\theta$ and $\phi$ ) such that $\sup _{\delta_{n}<|t| \leq \pi} m(t) \leq m\left(\delta_{n}\right)$ for all $n \in \mathbb{N}$ with $n>n_{0}$. Therefore

$$
\left|I_{2}\right| \leq \int_{\left\{\delta_{n}<|t| \leq \pi\right\}} e^{n \operatorname{Re}\left(g\left(z(\theta) e^{i t}\right)\right)} d t=\int_{\left\{\delta_{n}<|t| \leq \pi\right\}} e^{n m(t)} d t \leq 2 \pi e^{n m\left(\delta_{n}\right)} .
$$

The expansion $m(t)=g(z(\theta))-(z(\theta))^{2} g^{\prime \prime}(z(\theta)) t^{2} / 2+O\left(t^{4}\right)$ applied for $t=\delta_{n}$ yields $e^{n m\left(\delta_{n}\right)} \sim$ $e^{n g(z(\theta))} e^{-(z(\theta))^{2} g^{\prime \prime}(z(\theta)) n \delta_{n}^{2} / 2}$. Thus, $\left|I_{2}\right| / I_{1}=O\left(\sqrt{n} / e^{c n \delta_{n}^{2}}\right)$ with $c:=(z(\theta))^{2} g^{\prime \prime}(z(\theta)) / 2>0$. Since $n \delta_{n}^{2}=n^{1-2 \alpha} \rightarrow \infty$ it follows that $\left|I_{2}\right| / I_{1} \rightarrow 0$ as $n \rightarrow \infty$. Thus, the integral $I_{2}$ is negligible in comparison to $I_{1}$ and 23 is established.

For $k \in \mathbb{N}_{0}$ and $N \in \mathbb{N}$ with $N \geq k$ it follows from (21) that

$$
\begin{aligned}
\mathbb{P}\left(\mu_{N, 1}=k\right) & =\left(\begin{array}{c}
N \\
k
\end{array}\right) \frac{\sigma_{k}(\theta) \sigma_{N-k}((N-1) \theta)}{\sigma_{N}(N \theta)}=\frac{\sigma_{k}(\theta)}{k !} \frac{\frac{\sigma_{N-k}((N-l) \theta)}{(N-k) !}}{\frac{\sigma_{N}(N \theta)}{N !}} \\
& \sim \frac{\sigma_{k}(\theta)}{k !} \frac{(a(\theta))^{N} b_{k 1}(\theta) / \sqrt{2 \pi N}}{(a(\theta))^{N} b_{00}(\theta) / \sqrt{2 \pi N}}=\frac{\sigma_{k}(\theta)}{k !} \frac{b_{k 1}(\theta)}{b_{00}(\theta)}=\sigma_{k}(\theta) \frac{(z(\theta))^{k}}{k !} e^{-\theta \phi(z(\theta))} .
\end{aligned}
$$

Thus, $\mu_{N, 1} \rightarrow X$ in distribution as $N \rightarrow \infty$, where $X$ has distribution 15 .

In the following, for fixed $p \in(0, \infty)$, the convergence $\mathbb{E}\left(\mu_{N, 1}^{p}\right) \rightarrow \mathbb{E}\left(X^{p}\right)$ as $N \rightarrow \infty$ of the $p$-th moments is established. For all $N \in \mathbb{N}$ and all $k, l \in\{0, \ldots, N\}$ we have

$$
\begin{aligned}
& \frac{\sigma_{N-k}((N-l) \theta)}{(N-k) !} \leq \frac{\sigma_{N-k}(N \theta)}{(N-k) !}=\frac{1}{2 \pi i} \int_{C} z^{k-1} e^{N g(z)} d z \\
& \quad=\frac{1}{2 \pi i} \int_{-\pi}^{\pi}\left(z(\theta) e^{i t}\right)^{k-1} e^{N g\left(z(\theta) e^{i t}\right)} i z(\theta) e^{i t} d t=\frac{(z(\theta))^{k}}{2 \pi} \int_{-\pi}^{\pi} e^{i k t} e^{N g\left(z(\theta) e^{i t}\right)} d t .
\end{aligned}
$$

Taking the complex absolute value it follows for all $N \in \mathbb{N}$ and all $k, l \in\{0, \ldots, N\}$ that

$$
\frac{\sigma_{N-k}((N-l) \theta)}{(N-k) !} \leq \frac{(z(\theta))^{k}}{2 \pi} \int_{-\pi}^{\pi}\left|e^{N g\left(z(\theta) e^{i t}\right)}\right| d t=\frac{(z(\theta))^{k}}{2 \pi} \int_{-\pi}^{\pi} e^{N \operatorname{Re}\left(g\left(z(\theta) e^{i t}\right)\right)} d t .
$$

Since, by $23,(2 \pi)^{-1} \int_{-\pi}^{\pi} e^{N \operatorname{Re}\left(g\left(z(\theta) e^{i t}\right)\right)} d t \sim \sigma_{N}(N \theta) / N$ !, it follows that there exists a constant $N_{0} \in \mathbb{N}$ (which may depend on $\theta$ and $\phi$ but not on $k$ and $l$ ) such that

$$
\frac{\sigma_{N-k}((N-l) \theta)}{(N-k) !} \leq 2(z(\theta))^{k} \frac{\sigma_{N}(N \theta)}{N !}
$$


for all $N \geq N_{0}$ and all $k, l \in\{0, \ldots, N\}$. In particular, for all $N \geq N_{0}$ and all $k \in\{0, \ldots, N\}$,

$$
\mathbb{P}\left(\mu_{N, 1}=k\right)=\frac{\sigma_{k}(\theta)}{k !} \frac{\frac{\sigma_{N-k}((N-1) \theta)}{(N-k) !}}{\frac{\sigma_{N}(N \theta)}{N !}} \leq \frac{\sigma_{k}(\theta)}{k !} 2(z(\theta))^{k}=\kappa(\theta) \mathbb{P}(X=k),
$$

where $\kappa(\theta):=2 e^{\theta \phi(z(\theta))} \in(0, \infty)$. For all $p \in(0, \infty)$ the map $k \mapsto k^{p} \mathbb{P}(X=k), k \in \mathbb{N}_{0}$, is integrable with respect to the counting measure $\varepsilon_{\mathbb{N}_{0}}$ on $\mathbb{N}_{0}$, since $\int k^{p} \mathbb{P}(X=k) \varepsilon_{\mathbb{N}_{0}}(d k)=\sum_{k=0}^{\infty} k^{p} \mathbb{P}(X=k)=$ $\mathbb{E}\left(X^{p}\right)<\infty$. Dominated convergence yields the convergence $\mathbb{E}\left(\mu_{N, 1}^{p}\right) \rightarrow \mathbb{E}\left(X^{p}\right)$ as $N \rightarrow \infty$ of all moments.

In particular, $(N-1) c_{N}=\mathbb{E}\left(\left(\mu_{N, 1}\right)_{2}\right) \rightarrow \mathbb{E}\left((X)_{2}\right)>0$ and $(N-1)(N-2) d_{N}=\mathbb{E}\left(\left(\mu_{N, 1}\right)_{3}\right) \rightarrow$ $\mathbb{E}\left((X)_{3}\right)$ as $N \rightarrow \infty$. Thus, $d_{N} / c_{N}=O(1 / N) \rightarrow 0$ as $N \rightarrow \infty$, which ensures (see [18 or [16, Theorem 4 (b)]) that the considered symmetric compound Poisson model is in the domain of attraction of the Kingman coalescent. The proof is complete.

Proof 2. Let $X_{1}, X_{2}, \ldots$ be independent copies of the random variable $X$ with distribution (15). For $N \in \mathbb{N}$ define $S_{N}:=X_{1}+\cdots+X_{N}$. Since $0<\operatorname{Var}(X)<\infty$, the local limit theorem $\lim _{N \rightarrow \infty} \sup _{k \in \mathbb{Z}}\left|d_{N}(k)\right|=0$ holds, where $d_{N}(k):=\sqrt{N} \mathbb{P}\left(S_{N}=k\right)-g((k-N) / \sqrt{N})$ and $g$ denotes the density of the normal distribution $N(0, \operatorname{Var}(X))$. We have

$$
\mathbb{P}\left(\mu_{N, 1}=k\right)=\left(\begin{array}{c}
N \\
k
\end{array}\right) \frac{\sigma_{k}(\theta) \sigma_{N-k}((N-1) \theta)}{\sigma_{N}(N \theta)}=\mathbb{P}(X=k) \frac{\mathbb{P}\left(S_{N-1}=N-k\right)}{\mathbb{P}\left(S_{N}=N\right)} .
$$

Since $\mathbb{P}\left(S_{N}=N\right)=\left(g(0)+d_{N}(N)\right) / \sqrt{N} \sim g(0) / \sqrt{N}$ as $N \rightarrow \infty$ and, for arbitrary but fixed $k$, $\mathbb{P}\left(S_{N-1}=N-k\right)=\left(g((1-k) / \sqrt{N})+d_{N-1}(N-k)\right) / \sqrt{N-1} \sim g(0) / \sqrt{N}$ as $N \rightarrow \infty$ thanks to the fact that the convergence in the local limit theorem holds uniformly for all $k$, it follows that $\mathbb{P}\left(S_{N-1}=N-k\right) / \mathbb{P}\left(S_{N}=N\right) \rightarrow 1$ as $N \rightarrow \infty$ and, therefore, $\mathbb{P}\left(\mu_{N, 1}=k\right) \rightarrow \mathbb{P}(X=k)$ as $N \rightarrow \infty$ for all $k \in \mathbb{N}_{0}$. Thus, $\mu_{N, 1} \rightarrow X$ in distribution as $N \rightarrow \infty$. Fix $p \in(0, \infty)$. For all $N \in \mathbb{N}$,

$$
\mathbb{E}\left(\mu_{N, 1}^{p}\right)=\sum_{k=1}^{N} k^{p} \mathbb{P}(X=k) \frac{\mathbb{P}\left(S_{N-1}=N-k\right)}{\mathbb{P}\left(S_{N}=N\right)} .
$$

Applying the local limit theorem to both probabilities $\mathbb{P}\left(S_{N-1}=N-k\right)$ and $\mathbb{P}\left(S_{N}=N\right)$ it follows for arbitrary but fixed $p \in(0, \infty)$ that

$$
\begin{aligned}
\mathbb{E}\left(\mu_{N, 1}^{p}\right) & \sim \sum_{k=1}^{N} k^{p} \mathbb{P}(X=k) \frac{g((1-k) / \sqrt{N-1})+d_{N-1}(N-k)}{g(0)} \\
& \leq \sum_{k=1}^{N} k^{p} \mathbb{P}(X=k) \frac{g(0)+d_{N-1}(N-k)}{g(0)} \\
& \sim \sum_{k=1}^{N} k^{p} \mathbb{P}(X=k) \rightarrow \sum_{k=1}^{\infty} k^{p} \mathbb{P}(X=k)=\mathbb{E}\left(X^{p}\right),
\end{aligned}
$$

where we have used that the density $g$ is non-decreasing on $(-\infty, 0]$ and that $d_{N-1}(N-k) \rightarrow 0$ as $N \rightarrow \infty$ uniformly for all $k$ by the local limit theorem. Similarly,

$$
\mathbb{E}\left(\mu_{N, 1}^{p}\right) \geq \sum_{k=1}^{\left\lfloor N^{1 / 4}\right\rfloor} k^{p} \mathbb{P}(X=k) \frac{\mathbb{P}\left(S_{N-1}=N-k\right)}{\mathbb{P}\left(S_{N}=N\right)}
$$




$$
\begin{aligned}
& \sim \sum_{k=1}^{\left\lfloor N^{1 / 4}\right\rfloor} k^{p} \mathbb{P}(X=k) \frac{g((1-k) / \sqrt{N-1})+d_{N-1}(N-k)}{g(0)} \\
\geq & \sum_{k=1}^{\left\lfloor N^{1 / 4}\right\rfloor} k^{p} \mathbb{P}(X=k) \frac{g\left(\left(1-N^{1 / 4}\right) / \sqrt{N-1}\right)+d_{N-1}(N-k)}{g(0)} \\
\sim & \sum_{k=1}^{\left\lfloor N^{1 / 4}\right\rfloor} k^{p} \mathbb{P}(X=k) \rightarrow \sum_{k=1}^{\infty} k^{p} \mathbb{P}(X=k)=\mathbb{E}\left(X^{p}\right),
\end{aligned}
$$

where we used again that $g$ is non-decreasing on $(-\infty, 0]$ and that the convergence in the local limit theorem holds uniformly for all $k$. Thus, the convergence $\mathbb{E}\left(\mu_{N, 1}^{p}\right) \rightarrow \mathbb{E}\left(X^{p}\right)$ as $N \rightarrow \infty, p \in(0, \infty)$, is established. The rest of the proof is identical to the last four lines of Proof 1 .

Proof. (of Theorem 2.3) We verify that $\lim _{N \rightarrow \infty} c_{N}=0$. Fix $\varepsilon \in(0,1)$ and define $A_{i}:=\left\{\mu_{i} \leq N \varepsilon\right\}$ and $B_{i}:=\left\{\mu_{i}>N \varepsilon\right\}, i \in\{1, \ldots, N\}$. As in the proof of [13, Theorem 19.2], applied to the weights $w_{k}:=e^{-\theta \phi(r-)} \sigma_{k}(\theta) / k !$ and with $n:=m:=N$, it follows that there exists a constant $c_{\varepsilon} \in(-\infty, 0)$ such that $\mathbb{P}\left(\mu_{1}>N \varepsilon\right) \leq \exp \left(c_{\varepsilon} N+o(N)\right)$. In particular, $\lim _{N \rightarrow \infty} N \mathbb{P}\left(\mu_{1}>N \varepsilon\right)=0$ and

$$
\begin{aligned}
c_{N} & =\frac{1}{(N)_{2}} \sum_{i=1}^{N} \mathbb{E}\left(\left(\mu_{i}\right)_{2} 1_{A_{i}}\right)+\frac{1}{(N)_{2}} \sum_{i=1}^{N} \mathbb{E}\left(\left(\mu_{i}\right)_{2} 1_{B_{i}}\right) \leq \frac{N \varepsilon}{(N)_{2}} \sum_{i=1}^{N} \mathbb{E}\left(\mu_{i} 1_{A_{i}}\right)+\frac{N}{(N)_{2}} \mathbb{E}\left(\left(\mu_{1}\right)_{2} 1_{B_{1}}\right) \\
& \leq \frac{N \varepsilon}{(N)_{2}} \mathbb{E}\left(\mu_{1}+\cdots+\mu_{N}\right)+N \mathbb{E}\left(1_{B_{1}}\right)=\frac{N^{2} \varepsilon}{(N)_{2}}+N \mathbb{P}\left(\mu_{1}>N \varepsilon\right) \rightarrow \varepsilon, \quad N \rightarrow \infty .
\end{aligned}
$$

Thus, $\lim _{N \rightarrow \infty} c_{N}=0$, since $\varepsilon>0$ can be chosen arbitrarily small. In order to determine the asymptotics of the associated compound Poisson population model, let $\mu_{(1)} \geq \cdots \geq \mu_{(N)}$ denote the ranked offspring sizes, i.e. the offspring sizes $\mu_{1}, \ldots, \mu_{N}$, but permutated in non-increasing order. For all $\varepsilon>0$ we have $\mathbb{P}\left(\mu_{(1)}>N \varepsilon\right)=\mathbb{P}\left(\bigcup_{i=1}^{N}\left\{\mu_{i}>N \varepsilon\right\}\right) \leq N \mathbb{P}\left(\mu_{1}>N \varepsilon\right) \rightarrow 0$ as $N \rightarrow \infty$. Thus, $\mu_{(1)} / N \rightarrow 0$ in probability as $N \rightarrow \infty$. For arbitrary but fixed dimension $d \in \mathbb{N}$ it follows that $\left(\mu_{(1)} / N, \ldots, \mu_{(d)} / N\right) \rightarrow(0, \ldots, 0) \in \mathbb{R}^{d}$ in distribution as $N \rightarrow \infty$. Treating the infinite simplex $\Delta:=\left\{x=\left(x_{1}, x_{2}, \ldots\right): x_{1} \geq x_{2} \geq \cdots \geq 0, \sum_{i=1}^{\infty} x_{i} \leq 1\right\}$ as a subset of the metric space $\mathbb{R}^{\infty}$ equipped with the topology of pointwise convergence, this convergence of the finite-dimensional distributions is already equivalent (see, Billingsley [2, p. 19]) to the convergence of the full processes $\left(\mu_{(1)} / N, \ldots, \mu_{(N)} / N, 0,0, \ldots\right) \rightarrow(0,0, \ldots) \in \Delta$ in distribution as $N \rightarrow \infty$. Comparing this limit with Sagitov's convergence result [19, Theorem 2.1] shows that the model is in the domain of attraction of the Kingman coalescent.

Proof. (of Theorem 2.4 Let $X_{1}, X_{2}, \ldots$ be independent random variables all with the same distribution (16) of $X$. Define $S_{N}:=X_{1}+\cdots+X_{N}, N \in \mathbb{N}$. For all $k \in \mathbb{N}_{0}$ and all $N \geq k$,

$$
\mathbb{P}\left(\mu_{N, 1}=k\right)=\left(\begin{array}{l}
N \\
k
\end{array}\right) \frac{\sigma_{k}(\theta) \sigma_{N-k}((N-1) \theta)}{\sigma_{N}(N \theta)}=\mathbb{P}(X=k) \frac{\mathbb{P}\left(S_{N-1}=N-k\right)}{\mathbb{P}\left(S_{N}=N\right)} .
$$

The basic idea of the proof is to apply local limit theorems to the two probabilities $\mathbb{P}\left(S_{N-1}=N-k\right)$ and $\mathbb{P}\left(S_{N}=N\right)$ in the formula above. It turns out that the details of the proof differ for parts a), b) and c), so we have to treat these parts separately. 
a) By assumption, $\phi^{\prime \prime}(r-)<\infty$ or, equivalently, $\operatorname{Var}(X)<\infty$. Following the proof of Theorem 2.2 but with the root $z(\theta)$ replaced by $r$, it follows that $\mu_{N, 1} \rightarrow X$ in distribution as $N \rightarrow \infty$, where $X$ has distribution 116$)$. For $p \in(0,2]$ the convergence of moments $\mathbb{E}\left(\mu_{N, 1}^{p}\right) \rightarrow \mathbb{E}\left(X^{p}\right)$ as $N \rightarrow \infty$ is shown in the same way as in Proof 2 of Theorem 2.2 by exploiting the standard local limit theorem for sequences of independent and identically distributed random variables having finite and non-vanishing variance. In particular, $(N-1) c_{N}=\mathbb{E}\left(\left(\mu_{N, 1}\right)_{2}\right) \rightarrow \mathbb{E}\left((X)_{2}\right)=$ $\operatorname{Var}(X)=1+\theta r^{2} \phi^{\prime \prime}(r-)$ as $N \rightarrow \infty$. Thus, part a) of Theorem 2.4 is established.

b) Under the situation b) the local limit theorem $\lim _{N \rightarrow \infty} \sup _{k \in \mathbb{N}}\left|d_{N}(k)\right|=0$ holds, where $d_{N}(k)=\sqrt{N \log N} \mathbb{P}\left(S_{N}=k\right)-g((k-N) / \sqrt{N \log N}), N \in \mathbb{N}, k \in \mathbb{Z}$, and $g(x):=$ $(\pi c)^{-1 / 2} \exp \left(-x^{2} / c\right), x \in \mathbb{R}$, is the density of the normal distribution $N(0, c / 2)$. Applying this local limit theorem to both probabilities $\mathbb{P}\left(S_{N}=N\right)$ and $\mathbb{P}\left(S_{N-1}=N-k\right)$ it follows in the same way as in Proof 2 of Theorem 2.2 that $\mu_{N, 1} \rightarrow X$ in distribution as $N \rightarrow \infty$. Let us now turn to the asymptotics of the coalescence probability $c_{N}$. We have

$$
\begin{aligned}
(N-1) c_{N} & =\mathbb{E}\left(\left(\mu_{N, 1}\right)_{2}\right)=\sum_{k=2}^{N}(k)_{2} \mathbb{P}\left(\mu_{N, 1}=k\right) \\
& =\sum_{k=2}^{N}(k)_{2} \mathbb{P}(X=k) \frac{\mathbb{P}\left(S_{N-1}=N-k\right)}{\mathbb{P}\left(S_{N}=N\right)} \\
& \sim \sum_{k=2}^{N}(k)_{2} \mathbb{P}(X=k) \frac{g((1-k) / \sqrt{N \log N})+d_{N-1}(N-k)}{g(0)} \\
& \geq \sum_{k=2}^{\lfloor\sqrt{N}\rfloor}(k)_{2} \mathbb{P}(X=k) \frac{g\left((1-k) / \sqrt{N \log N)+d_{N-1}(N-k)}\right.}{g(0)} \\
& \geq \sum_{k=2}^{\lfloor\sqrt{N}\rfloor}(k)_{2} \mathbb{P}(X=k) \frac{g(-1 / \sqrt{\log N})+d_{N-1}(N-k)}{g(0)} \sim \sum_{k=2}^{\lfloor\sqrt{N}\rfloor}(k)_{2} \mathbb{P}(X=k) .
\end{aligned}
$$

Since $\mathbb{P}(X=k) \sim c k^{-3}$ as $k \rightarrow \infty$, the latter sum is asymptotically equal to $c \sum_{k=2}^{\lfloor\sqrt{N}\rfloor} 1 / k \sim$ $c \int_{2}^{\sqrt{N}} 1 / x d x \sim c \log \sqrt{N}=(c / 2) \log N$. Thus, $\liminf _{N \rightarrow \infty}\left(N c_{N}\right) / \log N \geq c / 2$. It remains to verify that $\lim \sup _{N \rightarrow \infty}\left(N c_{N}\right) / \log N \leq c / 2$. Define $B_{N}:=\lfloor\sqrt{N} \log N\rfloor, N \in \mathbb{N}$, and decompose the sum in (24) into a first part over all $k \leq B_{N}$ and a second part over all $k>B_{N}$. The second part is negligible, since, uniformly for all $k \in\left\{B_{N}+1, \ldots, N\right\}$,

$$
\begin{aligned}
\frac{\mathbb{P}\left(S_{N-1}=N-k\right)}{\mathbb{P}\left(S_{N}=N\right)} & \sim \frac{g\left((1-k) / \sqrt{N \log N)}+d_{N-1}(N-k)\right.}{g(0)} \\
& \leq \frac{g(-\sqrt{\log N})+d_{N-1}(N-k)}{g(0)} \rightarrow 0
\end{aligned}
$$

as $N \rightarrow \infty$. Note that we used that the normal density $g$ is non-decreasing on $(-\infty, 0]$, that $g(-\sqrt{\log N}) \rightarrow 0$ as $N \rightarrow \infty$ and that $d_{N-1}(N-k) \rightarrow 0$ as $N \rightarrow \infty$ uniformly for all $k$. For 
the first part we use $g(x) \leq g(0), x \in \mathbb{R}$, and obtain

$$
\begin{array}{rl}
\sum_{k=2}^{B_{N}}(k)_{2} & \mathbb{P}(X=k) \frac{\mathbb{P}\left(S_{N-1}=N-k\right)}{\mathbb{P}\left(S_{N}=N\right)} \\
& \sim \sum_{k=2}^{B_{N}}(k)_{2} \mathbb{P}(X=k) \frac{g((1-k) / \sqrt{N \log N})+d_{N-1}(N-k)}{g(0)} \\
& \leq \sum_{k=2}^{B_{N}}(k)_{2} \mathbb{P}(X=k) \frac{g(0)+d_{N-1}(N-k)}{g(0)} \sim \sum_{k=2}^{B_{N}}(k)_{2} \mathbb{P}(X=k),
\end{array}
$$

again thanks to the fact that the convergence in the local limit theorem holds uniformly for all $k$. From $\mathbb{P}(X=k) \sim c k^{-3}$ as $k \rightarrow \infty$ it follows that the last sum is asymptotically equal to $c \sum_{k=2}^{B_{N}} 1 / k \sim c \log B_{N} \sim(c / 2) \log N$. Thus, $\lim \sup _{N \rightarrow \infty}\left(N c_{N}\right) / \log N \leq c / 2$ and the asymptotics $c_{N} \sim(c / 2)(\log N) / N$ is established.

c) Let us first verify that $\mathbb{P}(X=k) \sim c k^{-\alpha-1}$ as $k \rightarrow \infty$ with $c:=\theta \kappa r^{\alpha} / \Gamma(-\alpha)$. It is readily checked that $(18)$ implies that

$$
\exp (\theta \phi(z))=A+B(z-r)+C(r-z)^{\alpha}+O\left((r-z)^{2}\right), \quad z \rightarrow r,
$$

with constants $A:=\exp (\theta \phi(r-)), B:=\theta \phi^{\prime}(r-) A$, and $C:=\theta \kappa A$. From that representation it follows that

$$
\begin{aligned}
P(X=k) & =r^{k} \exp (-\theta \phi(r-)) \frac{\sigma_{k}(\theta)}{k !}=\frac{r^{k}}{A}\left[z^{k}\right] \exp (\theta \phi(z)) \sim \frac{r^{k}}{A} C\left[z^{k}\right](r-z)^{\alpha} \\
& =\frac{r^{k}}{A} C \frac{r^{\alpha}}{r^{k}}\left(\begin{array}{l}
\alpha \\
k
\end{array}\right)(-1)^{k} \sim \frac{C}{A} r^{\alpha} \frac{1}{\Gamma(-\alpha) k^{\alpha+1}}=\frac{c}{k^{\alpha+1}}, \quad k \rightarrow \infty .
\end{aligned}
$$

By the generalized central limit theorem (see also Lemma 5.3 in the appendix), $\left(S_{N}-\right.$ $N) / N^{1 / \alpha} \rightarrow S$ in distribution as $N \rightarrow \infty$, where $S$ is an $\alpha$-stable random variable with characteristic function $\varphi(t):=\mathbb{E}(\exp (i t S))=\exp \left(c \Gamma(-\alpha)(-i t)^{\alpha}\right), t \in \mathbb{R}$. Define the constant $C:=-c \Gamma(-\alpha) \cos \frac{\pi \alpha}{2} \in(0, \infty)$ for convenience. For all $p \in[0, \infty)$,

$$
\begin{aligned}
\int_{-\infty}^{\infty}|t|^{p} & |\varphi(t)| d t \\
& =\int_{-\infty}^{\infty}|t|^{p} \exp \left(c \Gamma(-\alpha) \operatorname{Re}\left((-i t)^{\alpha}\right)\right) d t=\int_{-\infty}^{\infty}|t|^{p} \exp \left(c \Gamma(-\alpha)|t|^{\alpha} \cos \frac{\pi \alpha}{2}\right) d t \\
& =2 \int_{0}^{\infty} t^{p} \exp \left(-C t^{\alpha}\right) d t=\frac{2}{\alpha C^{(p+1) / \alpha}} \int_{0}^{\infty} u^{(p+1) / \alpha-1} \exp (-u) d u \\
& =\frac{2 \Gamma((p+1) / \alpha)}{\alpha C^{(p+1) / \alpha}} \in(0, \infty)
\end{aligned}
$$

where we have used the substitution $u=C t^{\alpha}\left(\Rightarrow d t / d u=1 /\left(\alpha C^{1 / \alpha}\right) u^{1 / \alpha-1}\right)$. In particular (choose $p=0$ ), $\varphi$ is integrable with respect to the Lebesgue measure on $\mathbb{R}$. Thus, by the 
Fourier inversion formula, $S$ has density

$$
\begin{aligned}
g(x) & =\frac{1}{2 \pi} \int_{-\infty}^{\infty} \exp \left(-i x t+c \Gamma(-\alpha)(-i t)^{\alpha}\right) d t \\
& =\frac{1}{\pi} \int_{0}^{\infty} \exp \left(c \Gamma(-\alpha) t^{\alpha} \cos \frac{\pi \alpha}{2}\right) \cos \left(x t+c \Gamma(-\alpha) t^{\alpha} \sin \frac{\pi \alpha}{2}\right) d t, \quad x \in \mathbb{R} .
\end{aligned}
$$

Note that $g$ is bounded, i.e. $\|g\|:=\sup _{x \in \mathbb{R}}|g(x)|<\infty$. Moreover (see, for example, Ibragimov and Linnik [12, Theorem 4.2.1]) the local limit theorem $\lim _{N \rightarrow \infty} \sup _{k \in \mathbb{Z}}\left|d_{N}(k)\right|=0$ holds, where $d_{N}(k):=N^{1 / \alpha} \mathbb{P}\left(S_{N}=k\right)-g\left((k-N) / N^{1 / \alpha}\right), N \in \mathbb{N}, k \in \mathbb{Z}$. In particular, $\mathbb{P}\left(S_{N}=\right.$ $N)=\left(g(0)+d_{N}(N)\right) / N^{1 / \alpha} \sim g(0) / N^{1 / \alpha}$ and

$$
\mathbb{P}\left(S_{N-1}=N-k\right)=\frac{g\left((1-k) /(N-1)^{1 / \alpha}\right)+d_{N-1}(N-k)}{(N-1)^{1 / \alpha}}
$$

The convergence $\mu_{N, 1} \rightarrow X$ in distribution therefore follows again in the same way as in Proof 2 of Theorem 2.2. We furthermore obtain

$$
\begin{aligned}
N c_{N} & \sim \mathbb{E}\left(\left(\mu_{N, 1}\right)_{2}\right) \sim \mathbb{E}\left(\mu_{N, 1}^{2}\right)=\sum_{k=1}^{N} k^{2} \frac{\mathbb{P}\left(X_{1}=k\right) \mathbb{P}\left(S_{N-1}=N-k\right)}{\mathbb{P}\left(S_{N}=N\right)} \\
& \sim \sum_{k=1}^{N} k^{2} \mathbb{P}(X=k) \frac{g\left((1-k) /(N-1)^{1 / \alpha}\right)+d_{N-1}(N-k)}{g(0)}=T_{1}+T_{2},
\end{aligned}
$$

where

$$
T_{1}:=\frac{1}{g(0)} \sum_{k=1}^{N} k^{2} \mathbb{P}(X=k) g\left((1-k) /(N-1)^{1 / \alpha}\right)
$$

and

$$
T_{2}:=\frac{1}{g(0)} \sum_{k=1}^{N} k^{2} \mathbb{P}(X=k) d_{N-1}(N-k) .
$$

Let us first analyse $T_{2}$. Since 18 holds by assumption, it follows that $\sup _{k \in \mathbb{Z}} \mid d_{N-1}(N-$ $k) \mid=O\left(N^{1-2 / \alpha}\right)$ by Theorem 5.4 (strong local limit theorem) in the appendix. Moreover, $\sum_{k=1}^{N} k^{2} \mathbb{P}(X=k) \sim c \sum_{k=1}^{N} k^{1-\alpha}=O\left(N^{2-\alpha}\right)$. Thus, $T_{2}=O\left(N^{1-2 / \alpha}\right) \sum_{k=1}^{N} k^{2} \mathbb{P}(X=k)=$ $O\left(N^{1-2 / \alpha}\right) O\left(N^{2-\alpha}\right)=O\left(N^{\delta}\right)$ with $\delta:=3-2 / \alpha-\alpha$. Let us now turn to $T_{1}$. Since the density $g$ is bounded, any finite number of values of the summation index $k$ in the sum of $T_{1}$ do not contribute to the asymptotics of $T_{1}$. It is therefore asymptotically allowed to replace $\mathbb{P}(X=k)$ by its asymptotic expression $c k^{-\alpha-1}$ and we obtain

$$
T_{1} \sim \frac{c}{g(0)} \sum_{k=1}^{N} k^{1-\alpha} g\left((1-k) /(N-1)^{1 / \alpha}\right) .
$$

For the last sum we have

$$
\sum_{k=1}^{N} k^{1-\alpha} g\left((1-k) /(N-1)^{1 / \alpha}\right)
$$




$$
\begin{aligned}
& =\sum_{k=1}^{N} N^{1 / \alpha} \int_{k / N^{1 / \alpha}}^{(k+1) / N^{1 / \alpha}}\left\lfloor x N^{1 / \alpha}\right\rfloor^{1-\alpha} g\left(\left(1-\left\lfloor x N^{1 / \alpha}\right\rfloor\right) /(N-1)^{1 / \alpha}\right) d x \\
& =N^{1 / \alpha} \int_{1 / N^{1 / \alpha}}^{(N+1) / N^{1 / \alpha}}\left\lfloor x N^{1 / \alpha}\right\rfloor^{1-\alpha} g\left(\left(1-\left\lfloor x N^{1 / \alpha}\right\rfloor\right) /(N-1)^{1 / \alpha}\right) d x \\
& \sim N^{1 / \alpha} \int_{0}^{\infty} x^{1-\alpha} N^{(1-\alpha) / \alpha} g(-x) d x=N^{(2-\alpha) / \alpha} \int_{0}^{\infty} x^{1-\alpha} g(-x) d x .
\end{aligned}
$$

Note that the last integral is finite, since $\int_{0}^{\infty} x^{1-\alpha} g(-x) d x \leq\|g\| \int_{0}^{1} x^{1-\alpha} d x+\int_{1}^{\infty} g(-x) d x \leq$ $\|g\| /(2-\alpha)+1<\infty$. Since $\delta=3-2 / \alpha-\alpha<(2-\alpha) / \alpha$, it follows in summary that $N c_{N} \sim T_{1}+T_{2} \sim T_{1}$, and (19) follows immediately. The proof of part c) is complete.

Proof. (of Theorem 2.5 Without loss of generality assume that $\phi$ has radius of convergence $r=1$. Otherwise consider the new power series $\phi^{*}$ defined via $\phi^{*}(z):=\phi(r z),|z|<1$, and note that the coefficients $\sigma_{k}^{*}(\theta)$ of the new power series $\phi^{*}$ and the coefficients $\sigma_{k}(\theta)$ of the original power series $\phi$ are related via $\sigma_{k}^{*}(\theta)=r^{k} \sigma_{k}(\theta), k \in \mathbb{N}_{0}, \theta \in(0, \infty)$.

Define $\Phi(z):=\Phi_{\theta}(z):=\exp (\theta \phi(z))$ for $|z|<1$ and $\theta \in(0, \infty)$. Note that $\left[z^{k}\right] \Phi(z)=\sigma_{k}(\theta) / k$ ! for $k \in \mathbb{N}_{0}$ and $\theta \in(0, \infty)$. By assumption there exist constants $c=c(\theta) \in(0, \infty)$ and $\beta=$ $\beta(\theta)>2$ such that $\sigma_{k}(\theta) / k ! \sim c k^{-\beta}$ as $k \rightarrow \infty$. We are hence in the situation to apply Janson's [13] condensation result (Theorem 19.34) to the weights $w_{k}:=\sigma_{k}(\theta) / k !, k \in \mathbb{N}_{0}$. Note that the weight sequence $\left(w_{k}\right)_{k \in \mathbb{N}_{0}}$ has $\operatorname{pgf} \sum_{k=0}^{\infty} w_{k} z^{k}=\exp (\theta \phi(z))=\Phi(z),|z|<1$. Moreover, in Janson's notation, $\Psi(z):=z \Phi^{\prime}(z) / \Phi(z)=\theta z \phi^{\prime}(z),|z|<1$, and, hence, $\nu:=\Psi(1-)=\theta \phi^{\prime}(1-)<1$ by assumption. Fix $k, l \in \mathbb{N}_{0}$. Applying [13, Theorem 19.34 (ii)] (with number of balls $m:=N-k$ and number of boxes $n:=N-l$ and $N \rightarrow \infty$, which implies $m / n \rightarrow 1=: \lambda>\nu)$ it follows for arbitrary but fixed $k, l \in \mathbb{N}_{0}$ and $\theta \in(0, \infty)$ that the partition function $Z(m, n)=\left[z^{m}\right] \Phi_{n \theta}(z)=\sigma_{m}(n \theta) / m$ ! satisfies

$$
\frac{\sigma_{N-k}((N-l) \theta)}{(N-k) !} \sim \frac{c}{(\lambda-\nu)^{\beta}} \frac{(\Phi(1-))^{N-l-1}}{N^{\beta-1}}
$$

as $N \rightarrow \infty$. For all $k \in \mathbb{N}_{0}$ and all $N \in \mathbb{N}$ with $N \geq k$ we have

$$
\mathbb{P}\left(\mu_{N, 1}=k\right)=\left(\begin{array}{c}
N \\
k
\end{array}\right) \frac{\sigma_{k}(\theta) \sigma_{N-k}((N-1) \theta)}{\sigma_{N}(N \theta)} .
$$

Using the asymptotics 27) for $l=0$ and for $l=1$ it follows that

$$
\lim _{N \rightarrow \infty} \mathbb{P}\left(\mu_{N, 1}=k\right)=\frac{\sigma_{k}(\theta)}{k !} \frac{1}{\Phi(1-)}=\frac{\sigma_{k}(\theta)}{k !} \exp (-\theta \phi(1-)), \quad k \in \mathbb{N}_{0},
$$

which is the right hand side of $\sqrt{16}$ for $r=1$. Thus, $\mu_{N, 1} \rightarrow X$ in distribution as $N \rightarrow \infty$.

As in the proof of Theorem 2.3 let $\mu_{(1)} \geq \cdots \geq \mu_{(N)}$ denote the ranked offspring sizes. Theorem 19.34 (i) of Janson [13] implies that

$$
\left(\mu_{(1)} / N, \ldots, \mu_{(N)} / N, 0,0, \ldots\right) \rightarrow(u, 0,0, \ldots)
$$

in distribution as $N \rightarrow \infty$, where $u:=\lambda-\nu=1-\theta \phi^{\prime}(1-) \in(0,1)$. 
In the following it is verified that $\lim _{N \rightarrow \infty} c_{N}=u^{2}$. We have $\mu_{(1)} / N \rightarrow u$ in distribution as $N \rightarrow \infty$. Since $\mu_{(1)} / N$ is bounded for all $N$ (between 0 and 1 ) it follows that $\mu_{(1)} / N \rightarrow u$ in $L^{2}$. In particular, $\mathbb{E}\left(\left(\mu_{(1)}\right)_{2}\right) /(N)_{2} \rightarrow u^{2}$ as $N \rightarrow \infty$. Since

$$
c_{N}=\frac{1}{(N)_{2}} \sum_{i=1}^{N} \mathbb{E}\left(\left(\mu_{(i)}\right)_{2}\right)=\frac{\mathbb{E}\left(\left(\mu_{(1)}\right)_{2}\right)}{(N)_{2}}+\frac{1}{(N)_{2}} \sum_{i=2}^{N} \mathbb{E}\left(\left(\mu_{(i)}\right)_{2}\right)
$$

it hence remains to verify that

$$
\lim _{N \rightarrow \infty} \frac{1}{(N)_{2}} \sum_{i=2}^{N} \mathbb{E}\left(\left(\mu_{(i)}\right)_{2}\right)=0 .
$$

In order to establish the convergence 300 fix $\varepsilon \in(0,1)$ and define $A:=\left\{\mu_{(2)} \leq N \varepsilon\right\}$ and its complement $B:=\left\{\mu_{(2)}>N \varepsilon\right\}$. We have

$$
\begin{aligned}
\frac{1}{(N)_{2}} \sum_{i=2}^{N} \mathbb{E}\left(\left(\mu_{(i)}\right)_{2}\right) & =\frac{1}{(N)_{2}} \sum_{i=2}^{N} \mathbb{E}\left(\left(\mu_{(i)}\right)_{2} 1_{A}\right)+\frac{1}{(N)_{2}} \sum_{i=2}^{N} \mathbb{E}\left(\left(\mu_{(i)}\right)_{2} 1_{B}\right) \\
& \leq \frac{N \varepsilon}{(N)_{2}} \sum_{i=2}^{N} \mathbb{E}\left(\mu_{(i)} 1_{A}\right)+\frac{N}{(N)_{2}} \sum_{i=2}^{N} \mathbb{E}\left(\mu_{(i)} 1_{B}\right) \\
& =\frac{N \varepsilon}{(N)_{2}} \mathbb{E}\left(\left(\mu_{(2)}+\cdots+\mu_{(N)}\right) 1_{A}\right)+\frac{N}{(N)_{2}} \mathbb{E}\left(\left(\mu_{(2)}+\cdots+\mu_{(N)}\right) 1_{B}\right) \\
& \leq \frac{N^{2} \varepsilon}{(N)_{2}}+\frac{N^{2}}{(N)_{2}} \mathbb{P}\left(\mu_{(2)}>N \varepsilon\right) \rightarrow \varepsilon, \quad \text { as } N \rightarrow \infty,
\end{aligned}
$$

since $\mathbb{P}\left(\mu_{(2)}>N \varepsilon\right) \rightarrow 0$ as $N \rightarrow \infty$. Since $\varepsilon>0$ can be chosen arbitrarily small, 30 holds and, hence, the convergence $\lim _{N \rightarrow \infty} c_{N}=u^{2} \in(0,1)$ is established. Comparing the limit (29) with Sagitov's convergence result [19, Theorem 2.2] it follows that the associated compound Poisson model is in the domain of attraction of a discrete-time $\Xi$-coalescent with $\Xi(d x) / \sum_{i=1}^{\infty} x_{i}^{2}=\delta_{(u, 0,0, \ldots)}(d x)$ being the Dirac measure at $(u, 0,0, \ldots) \in \Delta:=\left\{x=\left(x_{1}, x_{2}, \ldots\right): x_{1} \geq x_{2} \geq \cdots \geq 0, \sum_{i=1}^{\infty} x_{i} \leq 1\right\}$. Hence, the limiting process is a discrete-time coalescent with multiple collisions ( $\Lambda$-coalescent) with $\Lambda(d t) / t^{2}=\delta_{u}(d t)$ being the Dirac measure at $u$.

\section{Examples}

We start with the two most popular examples, the Wright-Fisher model and the Dirichlet model. Note that (6) holds for these two models. These two examples have the advantage that most calculations can be done explicitly. For example, we will verify the asymptotic results stated in Theorem 2.2 directly.

Example 4.1 (Wright-Fisher model) For the standard symmetric Wright-Fisher model, $\phi(z)=$ $z$. Therefore, $\xi_{n}$ has a Poisson distribution with parameter $\theta z$ and $\sigma_{k}(\theta)=\theta^{k}, k \in \mathbb{N}_{0}, \theta \in(0, \infty)$. For $k \in \mathbb{N}_{0}$ and $\theta \in(0, \infty)$ it follows that

$$
\mathbb{P}\left(\mu_{N, 1}=k\right)=\left(\begin{array}{c}
N \\
k
\end{array}\right) \frac{\sigma_{k}(\theta) \sigma_{N-k}((N-1) \theta)}{\sigma_{N}(N \theta)}=\left(\begin{array}{c}
N \\
k
\end{array}\right)\left(\frac{1}{N}\right)^{k}\left(1-\frac{1}{N}\right)^{N-k} .
$$


Thus, $\mu_{N, 1}$ has a binomial distribution with parameters $N$ and $1 / N$. In particular, for arbitrary but fixed $p \in \mathbb{N}, \mathbb{E}\left(\left(\mu_{N, 1}\right)_{p}\right)=(N)_{p} / N^{p} \rightarrow 1=\mathbb{E}\left((X)_{p}\right)$ as $N \rightarrow \infty$, where $X$ has a Poisson distribution with parameter 1 . The solution $z(\theta)$ of the equation $\theta z(\theta) \phi^{\prime}(z(\theta))=1$ is $z(\theta)=1 / \theta$. Note that (21) holds with $a(\theta):=e \theta$ and $b_{k l}(\theta):=\theta^{-k} e^{-l}, k, l \in \mathbb{N}_{0}, \theta \in(0, \infty)$. The effective population size $N_{e}=1 / c_{N}=N$ coincides with the actual population size $N$, a well known result.

Example 4.2 (Dirichlet model) For the symmetric Dirichlet model, $\phi(z)=-\log (1-z),|z|<1$. Therefore, $\xi_{1}$ has a negative binomial distribution with parameters $\theta$ and $1-z$. In particular, $\mathbb{E}\left(\xi_{1}\right)=\theta z \phi^{\prime}(z)=\theta z /(1-z)$. Moreover, $\sigma_{k}(\theta)=[\theta]_{k}:=\theta(\theta+1) \cdots(\theta+k-1)=\Gamma(k+\theta) / \Gamma(\theta)$, $k \in \mathbb{N}_{0}, \theta \in(0, \infty)$. Thus, for $k \in \mathbb{N}_{0}$ and $\theta \in(0, \infty)$,

$$
\mathbb{P}\left(\mu_{N, 1}=k\right)=\left(\begin{array}{c}
N \\
k
\end{array}\right) \frac{[\theta]_{k}[(N-1) \theta]_{N-k}}{[N \theta]_{N}}=\frac{[\theta]_{k}}{k !}(N)_{k} \frac{\Gamma(N \theta-\theta+N-k) \Gamma(N \theta)}{\Gamma(N \theta-\theta) \Gamma(N \theta+N)} .
$$

Since $\Gamma(x+c) \sim x^{c} \Gamma(x)$ as $x \rightarrow \infty$ for any $c \in \mathbb{R}$, it follows that

$$
\begin{aligned}
\mathbb{P}\left(\mu_{N, 1}=k\right) & \sim \frac{[\theta]_{k}}{k !}(N)_{k} \frac{(N \theta+N)^{-\theta-k} \Gamma(N \theta+N) \Gamma(N \theta)}{(N \theta)^{-\theta} \Gamma(N \theta) \Gamma(N \theta+N)} \\
& =\frac{[\theta]_{k}}{k !} \frac{(N)_{k}}{(N \theta+N)^{k}}\left(\frac{\theta+1}{\theta}\right)^{-\theta} \rightarrow \frac{[\theta]_{k}}{k !}\left(\frac{1}{\theta+1}\right)^{k}\left(\frac{\theta}{\theta+1}\right)^{\theta} .
\end{aligned}
$$

Thus $\mu_{N, 1} \rightarrow X$ in distribution as $N \rightarrow \infty$ where $X$ has a negative binomial distribution with parameters $\theta$ and $\theta /(\theta+1) \in(0,1)$. Moreover, for arbitrary but fixed $p \in \mathbb{N}, \mathbb{E}\left(\left(\mu_{N, 1}\right)_{p}\right)=$ $(N)_{p}[\theta]_{p} /[N \theta]_{p} \rightarrow[\theta]_{p} / \theta^{p}=\mathbb{E}\left((X)_{p}\right)$ as $N \rightarrow \infty$. Thus, $\mathbb{E}\left(\mu_{N, 1}^{p}\right) \rightarrow \mathbb{E}\left(X^{p}\right)$ as $N \rightarrow \infty$ for all $p \in \mathbb{N}$ in agreement with the general results derived in the proof of Theorem 2.2. The solution $z(\theta)$ of the equation $\theta z(\theta) \phi^{\prime}(z(\theta))=1$ is $z(\theta)=1 /(\theta+1)$. Note that 21$)$ holds with $a(\theta):=(\theta+1)^{\theta+1} / \theta^{\theta}$ and $b_{k l}(\theta):=(\theta /(\theta+1))^{l \theta+1 / 2}(1 /(\theta+1))^{k}, k, l \in \mathbb{N}_{0}, \theta \in(0, \infty)$. The symmetric Dirichlet model has effective population size $N_{e}=1 / c_{N}=(N-1) / \mathbb{E}\left(\left(\mu_{N, 1}\right)_{2}\right)=(N \theta+1) /(\theta+1) \sim \varrho N$ with $\varrho=\theta /(\theta+1)=1 / \operatorname{Var}(X)$, in agreement with Theorem 2.2 .

Let us now study examples which do not satisfy (6). We start with a model which involves the absolute Lah numbers.

Example 4.3 Suppose that $\phi_{m}=m$ ! for all $m \in \mathbb{N}$ or, equivalently, that $\phi(z)=z /(1-z)$, $|z|<1$. Note that $\phi^{\prime}(z)=1 /(1-z)^{2}$ and that $\phi^{\prime \prime}(z)=2 /(1-z)^{3}=2 \phi^{\prime}(z) /(1-z),|z|<1$. Then (Comtet [5, Section 3.3, p. 135, Theorem B]), $\sigma_{n}(\theta)=\sum_{k=1}^{n} L(n, k) \theta^{k}, n \in \mathbb{N}, \theta \in \mathbb{R}$, where $L(n, k):=B_{n k}(1 !, 2 !, \ldots)=n !(n-1) ! /(k !(k-1) !(n-k) !), n \in \mathbb{N}, k \in\{1, \ldots, n\}$, denote the absolute Lah numbers. The solution $z=z(\theta) \in(0,1)$ of the equation $1=\theta z \phi^{\prime}(z)=\theta z /(1-z)^{2}$ is $z(\theta)=1+\theta / 2-\sqrt{\theta(\theta+4)} / 2$. By Theorem 2.2. the model is in the domain of attraction of the Kingman coalescent and the effective population size $N_{e}$ satisfies $N_{e} \sim \varrho N$ with $\varrho:=1 /(1+$ $\left.\theta z^{2} \phi^{\prime \prime}(z)\right)=(1-z) /(1+z)<1$, since

$$
1+\theta z^{2} \phi^{\prime \prime}(z)=1+\theta z^{2} \phi^{\prime}(z) \frac{2}{1-z}=1+\frac{2 z}{1-z}=\frac{1+z}{1-z} .
$$

Note that the asymptotics 21 holds with $a(\theta)=e^{\theta \phi(z)} / z=e^{\theta z /(1-z)} / z=e^{1-z} / z$ and $b_{k l}(\theta):=$ $z^{k} e^{-l \theta \phi(z)} / \sqrt{1+\theta z^{2} \phi^{\prime \prime}(z)}=z^{k} e^{-l(1-z)} / \sqrt{(1+z) /(1-z)}, k, l \in \mathbb{N}_{0}, \theta \in(0, \infty)$. 
In the following example, which generalizes Example 4.3 , the series $\phi$ solves a particular functional equation and is hence (for most parameter constellations) only defined implicitly. Nevertheless an explicit expression for the solution $z=z(\theta)$ of the equation $\theta z \phi^{\prime}(z)=1$ is derived.

Example 4.4 Suppose that (see [1, p. 402, Eqs. (50) and (51)]) $\phi_{m}=(m-1) !\left(\begin{array}{c}a m \\ m-1\end{array}\right) b^{m-1}, m \in \mathbb{N}$, where it is assumed that the real parameters $a$ and $b$ are either both negative or $b>0$ and $a \geq 1$ (such that all the coefficients $\phi_{m}$ are non-negative). Note that [1, Eq. (43)] the power series $\phi(z)=\sum_{m=1}^{\infty} \phi_{m} z^{m} / m$ ! is the solution of the functional equation $\phi(z)=z f(\phi(z))$ with $f(x):=(1+b x)^{a}$ and that $\phi$ is related to the generalized binomial series $B_{a}$ (see, for example, [8, p. 200]) via $\phi(z)=\left(B_{a}(b z)-1\right) / b$. It is readily checked that $\phi$ has radius of convergence $r:=\lim _{m \rightarrow \infty}(m+1) \phi_{m} / \phi_{m+1}=(a b)^{-1}(1-1 / a)^{a-1} \in(0, \infty)$ and that $\phi(r-)=1 /(b(a-1))(=\infty$ for $a=1)$, since, for $a \neq 1, \phi(r-)=1 /(b(a-1))$ is the only positive solution of the equation $\phi(r-)=r f(\phi(r-))$. Differentiating both sides of the functional equation $\phi(z)=z f(\phi(z))$ yields the derivatives

$$
\phi^{\prime}(z)=\frac{f(\phi(z))}{1-z f^{\prime}(\phi(z))} \quad \text { and } \quad \phi^{\prime \prime}(z)=\frac{2 f^{\prime}(\phi(z)) \phi^{\prime}(z)+z f^{\prime \prime}(\phi(z))\left(\phi^{\prime}(z)\right)^{2}}{1-z f^{\prime}(\phi(z))}, \quad|z|<r .
$$

For $a=1$ (and $b>0$ ) we have $\phi_{m}=m ! b^{m-1}, m \in \mathbb{N}$, and $\phi(z)=z /(1-b z),|z|<1 / b$. For $a=b=1$ we are back in Example 4.3. For $a=2$ we have $\phi(z)=4 z /(1+\sqrt{1-4 b z})^{2}$, $|z|<1 /(4 b)$. For $a=-1$ (and $b<0)$ we have $\phi_{m}=(2 m-2) ! /(m-1) !(-b)^{m-1}, m \in \mathbb{N}$, and $\phi(z)=(\sqrt{1+4 b z}-1) /(2 b)=2 z /(1+\sqrt{1+4 b z}),|z|<1 /(-4 b)$. For general parameters $a$ and $b$, to the best of the authors knowledge, there seems to be no special function related to the power series $\phi$. Nevertheless, an explicit expression for the solution $z=z(\theta)$ of the equation $\theta z \phi^{\prime}(z)=1$ is obtained as follows. Let $x=x(\theta)$ be the solution in the open interval $(0,1)$ of the quadratic equation $\theta x=b(1-x)(a-x)$, i.e.

$$
x(\theta)=\frac{\theta+b+a b-\sqrt{(\theta+b+a b)^{2}-4 a b^{2}}}{2 b} \in(0,1) .
$$

We have $0<(1-x) / \theta<1 /(b(a-1))=\phi(r-)(=\infty$ for $a=1)$. Thus, $z=z(\theta):=\phi^{-1}((1-x) / \theta) \in$ $(0, r)$ is well defined. From $(1-x) / \theta=\phi(z)=z f(\phi(z))=z f((1-x) / \theta)$ it follows that

$$
\begin{aligned}
z & =\frac{1-x}{\theta} \frac{1}{f\left(\frac{1-x}{\theta}\right)}=\frac{1-x}{\theta} \frac{1}{\left(1+b \frac{1-x}{\theta}\right)^{a}}=\frac{1-x}{\theta} \frac{1}{\left(1+\frac{x}{a-x}\right)^{a}} \\
& =\frac{1-x}{\theta}\left(\frac{a-x}{a}\right)^{a}=\frac{1-x}{\theta} \frac{a-x}{a}\left(\frac{a-x}{a}\right)^{a-1}=\frac{x}{a b}\left(\frac{a-x}{a}\right)^{a-1} .
\end{aligned}
$$

Moreover,

$$
f^{\prime}(\phi(z))=f^{\prime}\left(\frac{1-x}{\theta}\right)=a b\left(1+b \frac{1-x}{\theta}\right)^{a-1}=a b\left(1+\frac{x}{a-x}\right)^{a-1}=a b\left(\frac{a}{a-x}\right)^{a-1}=\frac{x}{z}
$$

and, hence,

$$
\theta z \phi^{\prime}(z)=\frac{\theta z f(\phi(z))}{1-z f^{\prime}(\phi(z))}=\frac{\theta \phi(z)}{1-z f^{\prime}(\phi(z))}=\frac{1-x}{1-z \frac{x}{z}}=1 .
$$

Thus, $z=z(\theta)$ satisfies $\theta z \phi^{\prime}(z)=1$. Theorem 2.2 is therefore applicable. Straightforward computations show that the limiting random variable $X$ in Theorem 2.2 has variance $\operatorname{Var}(X)=$ 
$1+\theta z^{2} \phi^{\prime \prime}(z)=\left(a-x^{2}\right) /\left(a(1-x)^{2}\right)$. By Theorem 2.2 the model is in the domain of attraction of the Kingman coalescent and the effective population size $N_{e}$ satisfies $N_{e} \sim \varrho N$ as $N \rightarrow \infty$ with $\varrho=1 / \operatorname{Var}(X)=a(1-x)^{2} /\left(a-x^{2}\right) \in(0,1)$. Note that the asymptotics 21) holds, where $a(\theta)=e^{\theta \phi(z)} / z=e^{1-x} / z$ and

$$
b_{k l}(\theta)=\frac{z^{k} e^{-l \theta \phi(z)}}{\sqrt{1+\theta z^{2} \phi^{\prime \prime}(z)}}=z^{k} e^{-l(1-x)} \sqrt{\frac{a(1-x)^{2}}{a-x^{2}}}
$$

with $x=x(\theta)$ and $z=z(\theta)$ defined in 31$)$ and $(32)$. At first glance the solution $x(\theta)$ of the quadratic equation $\theta x=b(1-x)(a-x)$ seems to come 'from nowhere'. In the following an intuitive argument is provided showing how $x(\theta)$ comes into play. It is known [1, Eq. (51)] that $\sigma_{n}(\theta)=\sum_{j=1}^{n} B(n, j) \theta^{j}$ with

$$
B(n, j)=\frac{(n-1) !}{(j-1) !}\left(\begin{array}{c}
a n \\
n-j
\end{array}\right) b^{n-j}
$$

The fraction $\frac{B(n, n-j+1)(n \theta)^{n-j+1}}{B(n, n-j)(n \theta)^{n-j}}=\frac{n \theta j}{(n-j)(a n-j+1) b}$ is equal to 1 for $j=j_{n}$ with

$$
\begin{aligned}
j_{n} & :=\frac{n(\theta+b+a b)+b-\sqrt{(n(\theta+b+a b)+b)^{2}-4 b\left(b n+a b n^{2}\right)}}{2 b} \\
& \sim n \frac{\theta+b+a b-\sqrt{(\theta+b+a b)^{2}-4 a b^{2}}}{2 b}=n x(\theta) .
\end{aligned}
$$

Intuitively, when $n$ is large, the contribution to the sum $\sigma_{n}(n \theta)=\sum_{j=0}^{n-1} B(n, n-j)(n \theta)^{n-j}$ is essentially entirely originated from indices $j$ having the property that $j / n$ belongs to a (small) neighborhood of $x(\theta)$. The choice of the neighborhood is rather unimportant. It is hence not surprising that $x(\theta)$ plays a crucial role in finding the asymptotics of $\sigma_{n}(n \theta)$. Since the Bell numbers (33) are known explicitly, one may carry out the Laplace method in detail leading to an alternative proof of 21). We leave the details to the reader.

Example 4.5 Suppose that $\phi_{m}=1$ for all $m \in \mathbb{N}$ or, equivalently, that $\phi(z)=e^{z}-1, z \in \mathbb{C}$. Then (see, for example, Comtet [5, Section 3.3, p. 135, Theorem B]) $\sigma_{n}(\theta)=\sum_{k=1}^{n} S(n, k) \theta^{k}, n \in \mathbb{N}$, $\theta \in \mathbb{R}$, where the $S(n, k)$ denote the Stirling numbers of the second kind. In this case the solution $z=z(\theta)$ of the equation $1=\theta z \phi^{\prime}(z)=\theta z e^{z}$ cannot be expressed in closed form anymore. By Theorem 2.2, $\mu_{N, 1} \rightarrow X$ in distribution, where $X$ has distribution (15), and the model is in the domain of attraction of the Kingman coalescent. The effective population size $N_{e}$ satisfies $N_{e} \sim \varrho N$ with $\varrho:=1 /\left(1+\theta z^{2} \phi^{\prime \prime}(z)\right)=1 /\left(1+\theta z^{2} e^{z}\right)=1 /(1+z)<1$.

For two further examples where Theorem 2.2 is applicable we refer the reader to [10, Examples 1 and 2]. All examples considered so far satisfy $\phi^{\prime}(r-)=\infty$, which implies that for all $\theta \in(0, \infty)$ the equation $\theta z \phi^{\prime}(z)=1$ has a unique real solution $z=z(\theta) \in(0, r)$. Let us now study examples satisfying $\phi^{\prime}(r-)<\infty$.

Example 4.6 (Polylog model) Fix $\alpha \in(0, \infty)$ and suppose that $\phi$ is the polylog function, i.e. $\phi(z):=\sum_{m=1}^{\infty} m^{-\alpha} z^{m},|z|<1$. For $\alpha=1$ the polylog model coincides with the Dirichlet model (Example 4.2 with $\phi(z)=-\log (1-z)$ ). For $\alpha \rightarrow 0$ we are back in Example 4.3 whereas for $\alpha \rightarrow \infty$ we approach the Wright-Fisher model (Example 4.1). 
For non-integer parameter $\alpha \in(0, \infty) \backslash \mathbb{N}$, the first $\lfloor\alpha\rfloor-1$ derivatives of the polylog function $\phi$ are finite and $\phi$ has asymptotic expansion around $z=1$ of the form

$$
\phi(z)=\sum_{j=0}^{\lfloor\alpha\rfloor-1} \frac{\phi^{(j)}(1-)}{j !}(z-1)^{j}+\Gamma(1-\alpha)(1-z)^{\alpha-1}+O\left((1-z)^{\lfloor\alpha\rfloor}\right), \quad z \rightarrow 1,
$$

whereas for integer $\alpha \in \mathbb{N}$ the expansion has the form (see also [7, p. 411])

$$
\phi(z)=\sum_{j=0}^{\alpha-2} \frac{\phi^{(j)}(1-)}{j !}(z-1)^{j}+\frac{(-1)^{\alpha}}{(\alpha-1) !}(1-z)^{\alpha-1} \log (1-z)+O\left((1-z)^{\alpha-1}\right), \quad z \rightarrow 1 .
$$

In the following it is verified that, for all $\alpha, \theta \in(0, \infty)$, the distribution 16 satisfies

$$
\mathbb{P}(X=k)=\frac{\sigma_{k}(\theta)}{k !} \exp (-\theta \phi(1-)) \sim \frac{\theta}{k^{\alpha}}, \quad k \rightarrow \infty .
$$

Assume first that $2<\alpha<3$. Then, by (35), $\phi(z)=a+b(z-1)+\Gamma(1-\alpha)(1-z)^{\alpha-1}+O\left((1-z)^{2}\right)$ as $z \rightarrow 1$ with $a:=\phi(1-)=\zeta(\alpha)$ and $b:=\phi^{\prime}(1-)=\zeta(\alpha-1)$, where $\zeta$ denotes the Riemann zeta function. Thus,

$$
\Phi_{\theta}(z):=\exp (\theta \phi(z))=A+B(z-1)+C(1-z)^{\alpha-1}+O\left((1-z)^{2}\right), \quad z \rightarrow 1,
$$

where $A:=e^{\theta a}, B:=\theta b A$ and $C:=\theta \Gamma(1-\alpha) A$. Using

$$
\left[z^{k}\right](1-z)^{\alpha-1}=\left(\begin{array}{c}
\alpha-1 \\
k
\end{array}\right)(-1)^{k}=\frac{\Gamma(k-\alpha+1)}{\Gamma(1-\alpha) k !} \sim \frac{1}{\Gamma(1-\alpha) k^{\alpha}}, \quad k \rightarrow \infty,
$$

it follows that

$$
\mathbb{P}(X=k)=\exp (-\theta \phi(1-)) \frac{\sigma_{k}(\theta)}{k !}=\frac{1}{A}\left[z^{k}\right] \Phi_{\theta}(z) \sim \frac{C}{A}\left[z^{k}\right](1-z)^{\alpha-1} \sim \frac{\theta}{k^{\alpha}}, \quad k \rightarrow \infty,
$$

and (37) is established for $2<\alpha<3$. For arbitrary non-integer $\alpha \in(0, \infty)$ (37) follows from (35) similarly as shown above for $2<\alpha<3$. For integer $\alpha \in \mathbb{N}$ (37) follows from (36) and from [7, p. 387, Eq. (24)]. Thus, (37) holds for arbitrary $\alpha \in(0, \infty)$.

We now turn to the asymptotic behavior of the model as $N \rightarrow \infty$. If $\alpha \leq 2$, then $\phi^{\prime}(1-)=\infty$ and Theorem 2.2 is applicable for all $\theta \in(0, \infty)$, so the associated symmetric compound Poisson model is in the domain of attraction of the Kingman coalescent. There seems to be no closed expression available for the solution $z=z(\theta)$ of the equation $\theta z \phi^{\prime}(z)=1$. The case $\alpha=2$ is a nice exception where it is easily seen that $z(\theta)=1-e^{-1 / \theta}, \theta \in(0, \infty)$.

Assume now that $\alpha>2$. Then $\phi^{\prime}(1-)=\zeta(\alpha-1)<\infty$. In this case the equation $\theta z \phi^{\prime}(z)=1$ admits a solution $z=z(\theta) \in(0,1)$ if and only if $\theta>\theta_{c}$ with critical value $\theta_{c}:=1 / \phi^{\prime}(1-)<1$. Thus, Theorem 2.2 is not applicable for $\theta \leq \theta_{c}$. Note that $\phi^{\prime \prime}(1-)=\infty$ for $\alpha \leq 3$ and $\phi^{\prime \prime}(1-)=$ $\zeta(\alpha-2)-\zeta(\alpha-1)<\infty$ for $\alpha>3$.

If $\theta=\theta_{c}$ (critical case), then by Theorem 2.3, the model is still in the domain of attraction of the Kingman coalescent and, by Theorem 2.4 (part c) applied with $\alpha$ replaced by $\alpha-1$, so with 
constants $\kappa:=\Gamma(1-\alpha)$ and $c:=\theta_{c}$, the coalescence probability $c_{N}$ satisfies

$$
c_{N} \sim \begin{cases}\operatorname{Var}(X) / N & \text { if } \alpha \in(3, \infty), \\ \frac{\theta_{c}}{2} \frac{\log N}{N}=\frac{1}{2 \zeta(2)} \frac{\log N}{N}=\frac{3}{\pi^{2}} \frac{\log N}{N} & \text { if } \alpha=3, \\ \theta_{c} \frac{\int_{0}^{\infty} x^{2-\alpha} g(-x) d x}{g(0)} N^{2(2-\alpha) /(\alpha-1)} & \text { if } \alpha \in(2,3),\end{cases}
$$

where, in the first case $\alpha \in(3, \infty), \operatorname{Var}(X)=1+\theta_{c} \phi^{\prime \prime}(1-)=1+\phi^{\prime \prime}(1-) / \phi^{\prime}(1-)=1+(\zeta(\alpha-2)-$ $\zeta(\alpha-1)) / \zeta(\alpha-1)=\zeta(\alpha-2) / \zeta(\alpha-1) \in(1, \infty)$ and, in the third case $\alpha \in(2,3), g$ denotes the density of an $(\alpha-1)$-stable random variable with characteristic function $t \mapsto \exp \left(\theta_{c} \Gamma(1-\alpha)(-i t)^{\alpha-1}\right)$, $t \in \mathbb{R}$. Finally, if $0<\theta<\theta_{c}$ (subcritical case), then, by Theorem 2.5. the model is in the domain of attraction of a discrete-time $\Lambda$-coalescent with $\Lambda:=u^{2} \delta_{u}$ and $u:=1-\theta \phi^{\prime}(1-)=1-\theta \zeta(\alpha-1) \in$ $(0,1)$.

The following example is a slight modification of the polylog model. In comparison to the polylog model it has the advantage that for integer parameter $\alpha$ explicit expressions for the power series $\phi$ are available.

Example 4.7 (Modified polylog model) As in the polylog model, fix $\alpha \in(0, \infty)$, but suppose that $\phi(z)=\sum_{m=1}^{\infty} \Gamma(m) z^{m} / \Gamma(m+\alpha),|z|<1$. It does not come as a surprise that this model behaves very similar as the polylog model. We therefore leave the details to the reader and provide more information exemplary only for $\alpha=3$, in which case the explicit formula

$$
\phi(z)=\sum_{m=1}^{\infty} \frac{z^{m}}{m(m+1)(m+2)}=\frac{2(1-z)^{2}(-\log (1-z))-2 z+3 z^{2}}{4 z^{2}}, \quad|z|<1,
$$

is available. We have

$$
\phi^{\prime}(z)=\sum_{m=1}^{\infty} \frac{z^{m-1}}{(m+1)(m+2)}=\frac{2 z-z^{2}-2(1-z)(-\log (1-z))}{2 z^{3}}, \quad|z|<1
$$

and

$$
\phi^{\prime \prime}(z)=\sum_{m=2}^{\infty} \frac{(m-1) z^{m-2}}{(m+1)(m+2)}=\frac{z^{2}-6 z+2(3-2 z)(-\log (1-z))}{2 z^{4}}, \quad|z|<1 .
$$

Note that $\phi(1-)=1 / 4, \phi^{\prime}(1-)=1 / 2, \phi^{\prime \prime}(1-)=\infty$,

$$
\phi(z)=\frac{1}{4}+\frac{1}{2}(z-1)-\frac{1}{2}(1-z)^{2} \log (1-z)+O\left((1-z)^{2}\right), \quad z \rightarrow 1,
$$

and, for $\theta \in(0, \infty)$,

$$
\Phi_{\theta}(z):=\exp (\theta \phi(z))=A+B(z-1)-C(1-z)^{2} \log (1-z)+O\left((1-z)^{2}\right), \quad z \rightarrow 1,
$$

where $A:=\exp (\theta \phi(1-))=\exp (\theta / 4), B:=C:=\theta A / 2$. From $(1-z)^{2}(-\log (1-z))=(1-2 z+$ $\left.z^{2}\right) \sum_{k=1}^{\infty} z^{k} / k=z-(3 / 2) z^{2}+\sum_{k=3}^{\infty} 2 /(k(k-1)(k-2)) z^{k}$ it follows that $\left[z^{k}\right](1-z)^{2}(-\log (1-z))=$ $2 /(k(k-1)(k-2))$ for all $k \geq 3$ and, hence, $\left[z^{k}\right](1-z)^{2}(-\log (1-z)) \sim 2 / k^{3}$ as $k \rightarrow \infty$. Thus,

$\mathbb{P}(X=k):=\exp (-\theta \phi(1-)) \frac{\sigma_{k}(\theta)}{k !}=\frac{1}{A}\left[z^{k}\right] \Phi_{\theta}(z)=\frac{C}{A}\left[z^{k}\right](1-z)^{2}(-\log (1-z)) \sim \frac{2 C}{A k^{3}}=\frac{\theta}{k^{3}}$ 
as $k \rightarrow \infty$. If $\theta>1 / \phi^{\prime}(1-)=2$ (supercritical case), then, by Theorem 2.2 the associated compound Poisson model is in the domain of attraction of the Kingman coalescent and the coalescence probability $c_{N}$ satisfies $\lim _{N \rightarrow \infty} N c_{N}=1+\theta z^{2} \phi^{\prime \prime}(z)$, where $z$ is the solution of the equation $\theta z \phi^{\prime}(z)=1$ in the interval $(0,1)$.

If $\theta=2$ (critical case), then, by Theorem 2.3, the compound Poisson model is in the domain of attraction of the Kingman coalescent and, by (17), applied with constant $c:=\theta=2$, the coalescence probability satisfies $c_{N} \sim(\log N) / N$ as $N \rightarrow \infty$.

If $\theta \in(0,2)$ (subcritical case), then, by Theorem 2.5, the model is in the domain of attraction of a discrete-time $\Lambda$-coalescent with $\Lambda(d u):=u^{2} \delta_{u}$ and $u:=1-\theta \phi^{\prime}(1-)=1-\theta / 2 \in(0,1)$.

We provide another example satisfying $\phi^{\prime}(r-)<\infty$ and $\phi^{\prime \prime}(r-)=\infty$.

Example 4.8 (Lamperti model) Fix $\alpha \in(1,2)$ and $a \in(\alpha, \infty)$ and assume that $\phi(z)=a z-$ $1+(1-z)^{\alpha}$. Note that $\phi_{1}=a-\alpha>0$ and $\phi_{m}=(-1)^{m}(\alpha)_{m}>0$ for $m \in\{2,3, \ldots\}$. The series $\phi$ has radius of convergence $r=1$ with $\phi(1-)=a-1>0$. Moreover, $\phi^{\prime}(z)=a-\alpha(1-z)^{\alpha-1}$, $\phi^{\prime \prime}(z)=\alpha(\alpha-1)(1-z)^{\alpha-2}$, and $\phi^{\prime}(1-)=a \in(1, \infty)$ and $\phi^{\prime \prime}(1-)=\infty$. It is readily checked that

$$
\Phi_{\theta}(z):=\exp (\theta \phi(z))=A+B(z-1)+C(1-z)^{\alpha}+O\left((1-z)^{2}\right), \quad z \rightarrow 1,
$$

where $A:=\Phi_{\theta}(1-)=\exp (\theta(a-1)), B:=\Phi_{\theta}^{\prime}(1-)=\theta a A$ and $C:=\theta A$. Thus,

$$
\mathbb{P}(X=k):=\exp (-\theta \phi(1-)) \frac{\sigma_{k}(\theta)}{k !}=\frac{1}{A}\left[z^{k}\right] \Phi_{\theta}(z) \sim \frac{C}{A}\left[z^{k}\right](1-z)^{\alpha} \sim \frac{c}{k^{\alpha+1}}
$$

as $k \rightarrow \infty$, where $c:=C /(A \Gamma(-\alpha))=\theta / \Gamma(-\alpha)$. If $\theta a>1$ (supercritical case), then, by Theorem 2.2 , the associated symmetric compound Poisson population model is in the domain of attraction of the Kingman coalescent and the coalescence probability $c_{N}$ satisfies $c_{N} \sim\left(1+\theta z^{2} \phi^{\prime \prime}(z)\right) / N$ as $N \rightarrow \infty$, where $z=z(\theta)$ is the solution of the equation $\theta z \phi^{\prime}(z)=1$ in the interval $(0,1)$.

If $\theta a=1$ (critical case) then, by Theorem 2.3, the model is still in the domain of attraction of the Kingman coalescent, and, by Theorem 2.4 c), applied with constants $\kappa=1$ and $c=\theta / \Gamma(-\alpha)=$ $1 /(a \Gamma(-\alpha))$, the coalescence probability $c_{N}$ satisfies

$$
c_{N} \sim \frac{1}{a \Gamma(-\alpha)} \frac{\int_{0}^{\infty} x^{1-\alpha} g(-x) d x}{g(0)} N^{2(1-\alpha) / \alpha}, \quad N \rightarrow \infty
$$

with the density $g$ as defined in Theorem $2.4 \mathrm{c}$ ).

If $0<\theta a<1$ (subcritical case), then, by Theorem 2.5 the model is in the domain of attraction of a discrete-time $\Lambda$-coalescent with $\Lambda:=u^{2} \delta_{u}$ and $u:=1-\theta \phi^{\prime}(1-)=1-\theta a \in(0,1)$.

The following last example has much in common with the polylog model (Example 4.6).

Example 4.9 (exp polylog model) Fix $\alpha \in(0, \infty)$, let $Z(z):=\sum_{k=0}^{\infty} z^{k} /(k+1)^{\alpha}$ be the polylog function divided by $z$ and define $\phi(z):=\log (Z(z)),|z|<1$. Note that $\sigma_{k}(\theta) / k !=\left[z^{k}\right] \exp (\theta \phi(z))=$ $\left[z^{k}\right](Z(z))^{\theta} \geq \theta /(k+1)^{\alpha}$ for all $k \in \mathbb{N}$ and all $\theta \in(0, \infty)$. Therefore $\phi_{k}=\lim _{\theta \rightarrow 0} \sigma_{k}(\theta) / \theta \geq$ $k ! /(k+1)^{\alpha}>0$ for all $k \in \mathbb{N}$, so $\phi$ is a power series of the form $\phi(z)=\sum_{k=1}^{\infty} \phi_{k} z^{k} / k$ ! with strictly positive coefficients $\phi_{k}>0, k \in \mathbb{N}$. For $\alpha=1$ we have $\phi(z)=\log (-\log (1-z) / z)$ and for $\alpha \rightarrow 0$ we approach the Dirichlet model (Example 4.2 with $\phi(z)=-\log (1-z),|z|<1$.

For $2<\alpha<3$ we have

$$
Z(z)=\zeta(\alpha)+\zeta(\alpha-1)(z-1)+\Gamma(1-\alpha)(1-z)^{\alpha-1}+O\left((1-z)^{2}\right), \quad z \rightarrow 1 .
$$


Taking the $\theta$-power yields

$$
(Z(z))^{\theta}=(\zeta(\alpha))^{\theta}+\theta(\zeta(\alpha))^{\theta-1} \zeta(\alpha-1)(z-1)+\theta(\zeta(\alpha))^{\theta-1} \Gamma(1-\alpha)(1-z)^{\alpha-1}+O\left((1-z)^{2}\right)
$$

as $z \rightarrow 1$. Using standard singularity analysis (see Flajolet and Sedgewick [7, p. 385, Theorem VI.2] it follows that

$\mathbb{P}(X=k):=\exp (-\theta \phi(1-)) \frac{\sigma_{k}(\theta)}{k !}=(\zeta(\alpha))^{-\theta}\left[z^{k}\right] \exp (\theta \phi(z))=(\zeta(\alpha))^{-\theta}\left[z^{k}\right](Z(z))^{\theta} \sim \frac{\theta}{\zeta(\alpha) k^{\alpha}}$

as $k \rightarrow \infty$. The same asymptotic result for $\mathbb{P}(X=k)$ is checked similarly for arbitrary $\alpha \in(0, \infty)$. Let us now discuss the asymptotic behavior of the model. If $\alpha \leq 2$, then $\phi^{\prime}(1-)=\infty$, so Theorem 2.2 is applicable for all $\theta \in(0, \infty)$, which implies that the associated compound Poisson model is in the domain of attraction of the Kingman coalescent.

Assume now that $\alpha>2$. Then $\phi^{\prime}(1-)=\zeta(\alpha-1) / \zeta(\alpha)-1 \in(0, \infty)$. In this case the equation $\theta z \phi^{\prime}(z)=1$ admits a solution $z=z(\theta) \in(0,1)$ if and only if $\theta>\theta_{c}$ with critical value $\theta_{c}:=$ $1 / \phi^{\prime}(1-) \in(0, \infty)$. Thus, Theorem 2.2 is not applicable for $\theta \leq \theta_{c}$.

If $\theta=\theta_{c}$ (critical case), then, by Theorem 2.3. the model is still in the domain of attraction of the Kingman coalescent and, by Theorem 2.4 part c) applied with $\alpha$ replaced by $\alpha-1$, so with constants $\kappa:=\Gamma(1-\alpha) / \zeta(\alpha)$ and $c:=\theta_{c} / \zeta(\alpha)=1 /\left(\phi^{\prime}(1-) \zeta(\alpha)\right)=1 /(\zeta(\alpha-1)-\zeta(\alpha))$, the coalescence probability $c_{N}$ satisfies

$$
c_{N} \sim \begin{cases}\frac{1}{\zeta(\alpha-1)-\zeta(\alpha)} \frac{\int_{0}^{\infty} x^{2-\alpha} g(-x) d x}{g(0)} N^{2(2-\alpha) /(\alpha-1)} & \text { if } \alpha \in(2,3), \\ \frac{1}{2(\zeta(2)-\zeta(3))} \frac{\log N}{N} \approx 1.128981 \frac{\log N}{N} & \text { if } \alpha=3, \\ \operatorname{Var}(X) / N & \text { if } \alpha \in(3, \infty),\end{cases}
$$

where, in the first case $\alpha \in(2,3), g$ denotes the density of an $(\alpha-1)$-stable random variable with characteristic function $t \mapsto \exp \left(\theta_{c} \Gamma(1-\alpha)(-i t)^{\alpha-1}\right), t \in \mathbb{R}$, and in the third case $\alpha \in(3, \infty)$,

$$
\operatorname{Var}(X)=1+\theta_{c} \phi^{\prime \prime}(1-)=1+\frac{\phi^{\prime \prime}(1-)}{\phi^{\prime}(1-)}=\frac{\zeta(\alpha) \zeta(\alpha-2)-(\zeta(\alpha-1))^{2}}{\zeta(\alpha)(\zeta(\alpha-1)-\zeta(\alpha))},
$$

since $Z^{\prime \prime}(1-)=\zeta(\alpha-2)-3 \zeta(\alpha-1)+2 \zeta(\alpha)$ and, hence,

$$
\phi^{\prime \prime}(1-)=\frac{Z^{\prime \prime}(1-) Z(1-)-\left(Z^{\prime}(1-)\right)^{2}}{(Z(1-))^{2}}=\frac{\zeta(\alpha) \zeta(\alpha-2)-\zeta(\alpha) \zeta(\alpha-1)+(\zeta(\alpha))^{2}-(\zeta(\alpha-1))^{2}}{(\zeta(\alpha))^{2}} .
$$

If $0<\theta<\theta_{c}$ (subcritical case), then, by Theorem 2.5 the associated symmetric compound Poisson model is in the domain of attraction of a discrete-time $\Lambda$-coalescent with $\Lambda:=u^{2} \delta_{u}$ and $u:=$ $1-\theta \phi^{\prime}(1-)=1-\theta(\zeta(\alpha-1) / \zeta(\alpha)-1) \in(0,1)$.

\section{Appendix}

The following simple but useful lemma is needed in the proof of Theorem 2.2 .

Lemma 5.1 Let $a_{1}, a_{2}, \ldots \in[0, \infty)$ such that $\sum_{m=1}^{\infty} a_{m} q^{m}<\infty$ for some $q \in(1, \infty)$. Define $f(t):=\sum_{m=1}^{\infty} a_{m} \cos (m t), t \in \mathbb{R}$. If $a_{1}>0$ then there exists $\varepsilon_{0}>0$ such that $\sup _{\varepsilon<|t| \leq \pi} f(t) \leq f(\varepsilon)$ for all $\varepsilon \in\left[0, \varepsilon_{0}\right]$. 
Proof. Since $f(t)=f(-t)$ for $t \in \mathbb{R}$ it suffices to find some $\varepsilon_{0}>0$ such that $\sup _{\varepsilon<t<\pi} f(t) \leq$ $f(\varepsilon)$ for all $\varepsilon \in\left[0, \varepsilon_{0}\right]$. Obviously, $f(0)-f(t)=\sum_{m=1}^{\infty} a_{m}(1-\cos (m t)) \geq a_{1}(1-\cos t)>0$ for $t \in(0,2 \pi)$, so $f(t)<f(0)$ for $t \in(0,2 \pi)$. For arbitrary but fixed $t \in(-\infty, \log q)$, the map $(m, k) \mapsto a_{m}(-1)^{k}(m t)^{2 k} /(2 k)$ ! is integrable with respect to the counting measure $\varepsilon_{\mathbb{N}} \otimes \varepsilon_{\mathbb{N}_{0}}$ on $\mathbb{N} \times \mathbb{N}_{0}$, since

$$
\sum_{m=1}^{\infty} \sum_{k=0}^{\infty}\left|a_{m}(-1)^{k} \frac{(m t)^{2 k}}{(2 k) !}\right| \leq \sum_{m=1}^{\infty} a_{m} \sum_{l=0}^{\infty} \frac{(m t)^{l}}{l !}=\sum_{m=1}^{\infty} a_{m} e^{m t} \leq \sum_{m=1}^{\infty} a_{m} q^{m}<\infty
$$

by assumption. Thus, by Fubini's theorem,

$$
f(t)=\sum_{m=1}^{\infty} a_{m} \cos (m t)=\sum_{m=1}^{\infty} a_{m} \sum_{k=0}^{\infty}(-1)^{k} \frac{(m t)^{2 k}}{(2 k) !}=\sum_{k=0}^{\infty}(-1)^{k} \frac{t^{2 k}}{(2 k) !} \sum_{m=1}^{\infty} m^{2 k} a_{m} .
$$

so on the interval $(-\infty, \log q)$ the function $f$ is a power series in $t$. Together with $f^{\prime}(0)=0$ and $f^{\prime \prime}(0)=-\sum_{m=1}^{\infty} m^{2} a_{m}<0$ it follows that there exists a constant $\delta \in(0, \pi)$ such that $f$ is nonincreasing on $[0, \delta]$. The continuous function $f$ takes its supremum on the compact set $[\delta, \pi]$, so $\sup _{t \in[\delta, \pi]} f(t)=f\left(t_{0}\right)$ for some $t_{0} \in[\delta, \pi]$. Now choose $\varepsilon_{0} \in(0, \delta)$ sufficiently small such that $f\left(\varepsilon_{0}\right)>f\left(t_{0}\right)$, which is possible since $f$ is continuous and $f(0)>f\left(t_{0}\right)$. Now let $\varepsilon \in\left[0, \varepsilon_{0}\right]$ arbitrary but fixed. For $t \in(\varepsilon, \delta]$ we obtain $f(t) \leq f(\varepsilon)$, since $f$ is non-increasing on $[\varepsilon, \delta]$. For $t \in[\delta, \pi]$ we have $f(t) \leq f\left(t_{0}\right)<f\left(\varepsilon_{0}\right) \leq f(\varepsilon)$, since $f$ is non-increasing on $\left[\varepsilon, \varepsilon_{0}\right]$. Thus $\sup _{\varepsilon<t \leq \pi} f(t) \leq f(\varepsilon)$.

In the following we provide some results for certain $\alpha$-stable distributions, which, to the best of the authors knowledge, are not stated or at least quite hard to find in the literature. Our first result concerns half-sided moments.

Lemma 5.2 Fix $d>0$ and $1<\alpha<2$. Let $S$ be an $\alpha$-stable random variable with characteristic function $t \mapsto \exp \left(d(-i t)^{\alpha}\right), t \in \mathbb{R}$, and let $g$ denote the density of $S$. Then, for all $\beta \in(-1,0)$,

$$
\mathbb{E}\left((-S)^{\beta} 1_{\{S \leq 0\}}\right)=\int_{0}^{\infty} x^{\beta} g(-x) d x=d^{\beta / \alpha} \frac{\Gamma(\beta)}{\Gamma(\beta / \alpha)} .
$$

Moreover, $\mathbb{P}(S \leq 0)=1 / \alpha$ and $g(0)=d^{-1 / \alpha}|\Gamma(-1 / \alpha)|^{-1}$.

Proof. Since $S$ has the same distribution as $d^{1 / \alpha} S_{0}$, where $S_{0}$ is a random variable with characteristic function $t \mapsto \exp \left((-i t)^{\alpha}\right), t \in \mathbb{R}$, we may assume without loss of generality that $d=1$. By the Fourier inversion formula,

$$
\begin{aligned}
\int_{0}^{\infty} x^{\beta} g(-x) d x & =\int_{0}^{\infty} x^{\beta} \frac{1}{2 \pi} \int_{-\infty}^{\infty} \exp \left(i t x+(-i t)^{\alpha}\right) d t d x \\
& =\frac{1}{2 \pi} \int_{-\infty}^{\infty} \exp \left((-i t)^{\alpha}\right) \int_{0}^{\infty} x^{\beta} \exp (i t x) d x d t \\
& =\frac{\Gamma(\beta+1)}{2 \pi} \int_{-\infty}^{\infty} \exp \left((-i t)^{\alpha}\right)(-i t)^{-\beta-1} d t \\
& =\frac{\Gamma(\beta+1)}{2 \pi} \frac{\Gamma(-\beta / \alpha)}{\alpha} 2 \sin (-\pi \beta / \alpha) \\
& =\frac{\Gamma(\beta+1)}{\alpha \Gamma(1+\beta / \alpha)}=\frac{\Gamma(\beta)}{\Gamma(\beta / \alpha)},
\end{aligned}
$$


where the second last equality follows from Euler's reflection formula $\sin (\pi z) \Gamma(z)=\pi / \Gamma(1-z)$, applied to the point $z:=-\beta / \alpha<1 / \alpha<1$. The relation $\mathbb{P}(S \leq 0)=1 / \alpha$ follows by taking the limit $\beta \rightarrow 0$ in the second last fraction above. Similarly,

$$
g(0)=\frac{1}{2 \pi} \int_{-\infty}^{\infty} \exp \left((-i t)^{\alpha}\right) d t=\frac{1}{2 \pi} \frac{\Gamma(1 / \alpha)}{\alpha} 2 \sin (\pi / \alpha)=\frac{1}{\alpha \Gamma(1-1 / \alpha)}=|\Gamma(-1 / \alpha)|^{-1} .
$$

Let $X_{1}, X_{2}, \ldots$ be independent copies of a random variable $X$ having distribution (16). We provide a weak limiting result for $S_{N}:=X_{1}+\cdots+X_{N}, N \in \mathbb{N}$, which holds under the asymptotic assumption (18) on the power series $\phi$. The result provides detailed information on the rate of convergence in terms of the characteristic functions. As an application (see Theorem 5.4) a local limit theorem is obtained which provides information on the convergence rate.

Lemma 5.3 Assume that $\theta r \phi^{\prime}(r-)=1$ (critical case). If there exist constants $\alpha \in(1,2)$ and $\kappa=\kappa(\alpha)>0$ such that the power series $\phi$ of the considered compound Poisson model satisfies (18), then $\left(S_{N}-N\right) / N^{1 / \alpha} \rightarrow S$ in distribution as $N \rightarrow \infty$, where $S$ is an $\alpha$-stable random variable with characteristic function $\varphi(t):=\exp \left(\theta \kappa r^{\alpha}(-i t)^{\alpha}\right), t \in \mathbb{R}$. Moreover, there exists a constant $C>0$ such that the difference of the characteristic functions $\varphi_{\left(S_{N}-N\right) / N^{1 / \alpha}}$ of $\left(S_{N}-N\right) / N^{1 / \alpha}$ and $S$ satisfies

$$
\left|\varphi_{\left(S_{N}-N\right) / N^{1 / \alpha}}(t)-\varphi(t)\right| \leq|\varphi(t)|\left(e^{C t^{2} N^{1-2 / \alpha}}-1\right)
$$

for all $N \in \mathbb{N}$ and all $t \in \mathbb{R}$.

Proof. (of Lemma 5.3 Let $t \in \mathbb{R}$. By (18), the characteristic function $\varphi_{X}$ of the random variable $X$ with distribution (16) satisfies

$$
\begin{aligned}
\frac{\log \varphi_{X}(t)}{\theta} & =\phi\left(r e^{i t}\right)-\phi(r-)=\phi^{\prime}(r-)\left(r e^{i t}-r\right)+\kappa\left(r-r e^{i t}\right)^{\alpha}+O\left(\left(r-r e^{i t}\right)^{2}\right) \\
& =r \phi^{\prime}(r-)\left(e^{i t}-1\right)+\kappa r^{\alpha}\left(1-e^{i t}\right)^{\alpha}+O\left(r^{2}\left(1-e^{i t}\right)^{2}\right) \\
& =r \phi^{\prime}(r-)\left(i t+O\left(t^{2}\right)\right)+\kappa r^{\alpha}\left(-i t+O\left(t^{2}\right)\right)+O\left(t^{2}\right) \\
& =r \phi^{\prime}(r-) i t+\kappa r^{\alpha}(-i t)^{\alpha}+O\left(t^{2}\right), \quad t \rightarrow 0 .
\end{aligned}
$$

For convenience, define $B_{N}:=N^{1 / \alpha}$ for all $N \in \mathbb{N}$. The characteristic function of $\left(S_{N}-N\right) / B_{N}$ satisfies $\mathbb{E}\left(e^{i t\left(S_{N}-N\right) / B_{N}}\right)=e^{-i t N / B_{N}}\left(\varphi_{X}\left(t / B_{N}\right)\right)^{N}$ and, therefore,

$$
\begin{aligned}
& \log \mathbb{E}\left(e^{i t\left(S_{N}-N\right) / B_{N}}\right)=-i t N / B_{N}+N \log \varphi_{X}\left(t / B_{N}\right) \\
& \quad=-i t N / B_{N}+N \theta\left(r \phi^{\prime}(r-) i t / B_{N}+\kappa r^{\alpha}\left(-i t / B_{N}\right)^{\alpha}+O\left(\left(t / B_{N}\right)^{2}\right)\right) \\
& \quad=-i t N / B_{N}\left(1-\theta r \phi^{\prime}(r-)\right)+\theta \kappa r^{\alpha}(-i t)^{\alpha}+O\left(t^{2} N / B_{N}^{2}\right) \\
& \quad=\theta \kappa r^{\alpha}(-i t)^{\alpha}+O\left(t^{2} N / B_{N}^{2}\right)=\log \varphi(t)+O\left(t^{2} N / B_{N}^{2}\right) .
\end{aligned}
$$

Note that we have used that $\theta r \phi^{\prime}(r-)=1$ (critical case). Thus, for all $t \in \mathbb{R}$,

$$
\mathbb{E}\left(e^{i t\left(S_{N}-N\right) / B_{N}}\right)=e^{\log \varphi(t)+O\left(t^{2} N / B_{N}^{2}\right)}=\varphi(t) e^{O\left(t^{2} N / B_{N}^{2}\right)},
$$

where the constant of the O-term so far does not depend on $t$ and $N$, which implies that (38) holds. For arbitrary but fixed $t$ we in particular obtain

$$
\mathbb{E}\left(e^{i t\left(S_{N}-N\right) / B_{N}}\right)=\varphi(t)\left(1+O\left(N / B_{N}^{2}\right)=\varphi(t)+O\left(N / B_{N}^{2}\right) .\right.
$$


Note that $N / B_{N}^{2}=N^{1-2 / \alpha} \rightarrow 0$ as $N \rightarrow \infty$. By the continuity theorem it follows that $\left(S_{N}-\right.$ $N) / N^{1 / \alpha} \rightarrow S$ in distribution, where $S$ has characteristic function $\varphi$.

We are now able to verify a local limit theorem which provides information on the rate of convergence.

Theorem 5.4 (strong local limit theorem) Assume that the conditions of Lemma 5.3 are satisfied. Then, the local limit theorem

$$
\sup _{k \in \mathbb{Z}}\left|d_{N}(k)\right|=O\left(N^{1-2 / \alpha}\right)
$$

holds, where $d_{N}(k):=\left|N^{1 / \alpha} \mathbb{P}\left(S_{N}=k\right)-g\left((k-N) / N^{1 / \alpha}\right)\right|$ and $g$ denotes the density of the limiting $\alpha$-stable random variable $S$ defined in Lemma 5.3 .

Proof. (of Theorem 5.4 We modify the proof of the local limit theorem for lattice distributions of Ibragimov and Linnik [12, Theorem 4.2.1]. By the Fourier inversion formula, $S$ has density $g(x)=(2 \pi)^{-1} \int_{-\infty}^{\infty} e^{-i t x} \varphi(t) d t, x \in \mathbb{R}$. As in the proof of Lemma 5.3 define $B_{N}:=N^{1 / \alpha}, N \in \mathbb{N}$. The discrete inversion formula ensures that the integer valued random variable $S_{N}:=X_{1}+\cdots+X_{N}$ has distribution

$$
\begin{aligned}
P\left(S_{N}=k\right) & =\frac{1}{2 \pi} \int_{-\pi}^{\pi} e^{-i u k} \varphi_{S_{N}}(u) d u=\frac{1}{2 \pi} \int_{-\pi}^{\pi} e^{-i u k}\left(\varphi_{X}(u)\right)^{N} d u \\
& =\frac{1}{2 \pi B_{N}} \int_{-\pi B_{N}}^{\pi B_{N}} e^{-i t k / B_{N}}\left(\varphi_{X}\left(t / B_{N}\right)\right)^{N} d t \\
& =\frac{1}{2 \pi B_{N}} \int_{-\pi B_{N}}^{\pi B_{N}} e^{-i t\left(x+N / B_{N}\right)}\left(\varphi_{X}\left(t / B_{N}\right)\right)^{N} d t, \quad k \in \mathbb{Z},
\end{aligned}
$$

where we have used the substitution $u=t / B_{N}$ and where we use the notation $x:=(k-N) / B_{N}$. Thus,

$$
\begin{aligned}
d_{N}(k) & =\left|B_{N} \mathbb{P}\left(S_{N}=k\right)-g(x)\right| \\
& =\frac{1}{2 \pi}\left|\int_{-\pi B_{N}}^{\pi B_{N}} e^{-i t x} e^{-i t N / B_{N}}\left(\varphi_{X}\left(t / B_{N}\right)\right)^{N}-\int_{-\infty}^{\infty} e^{-i t x} \varphi(t) d t\right| \\
& \leq \int_{-\pi B_{N}}^{\pi B_{N}}\left|e^{-i t N / B_{N}}\left(\varphi_{X}\left(t / B_{N}\right)\right)^{N}-\varphi(t)\right| d t+\int_{|t|>\pi B_{N}}|\varphi(t)| d t \\
& \leq I_{1}+I_{2}+I_{3}+I_{4},
\end{aligned}
$$

with

$$
\begin{aligned}
I_{1} & :=\int_{-k_{N}}^{k_{N}}\left|e^{-i t N / B_{N}}\left(\varphi_{X}\left(t / B_{N}\right)\right)^{N}-\varphi(t)\right| d t \\
I_{2} & :=\int_{k_{N} \leq|t| \leq \varepsilon B_{N}}\left|\varphi_{X}\left(t / B_{N}\right)\right|^{N} d t \\
I_{3} & :=\int_{\varepsilon B_{N} \leq|t| \leq \pi B_{N}}\left|\varphi_{X}\left(t / B_{N}\right)\right|^{N} d t, \quad \text { and } \\
I_{4} & :=\int_{|t|>k_{N}}|\varphi(t)| d t,
\end{aligned}
$$


where we choose $k_{N}:=B_{N} / \sqrt{N}=N^{1 / \alpha-1 / 2}$ and where $\varepsilon \in(0, \pi)$ is a constant, to be determined. Note that the integrals $I_{j}, j \in\{1,2,3,4\}$, coincide with those on p. 122 of [12], except for the crucial modification that the constant $A$ on p. 122 in [12] is replaced by $k_{N}$ satisfying $k_{N} \geq 1$ for all $N \in \mathbb{N}$ and $k_{N} \rightarrow \infty$ as $N \rightarrow \infty$. We now turn to the estimation of these integrals and will show that $I_{1}=O\left(N^{1-2 / \alpha}\right)$ and that $I_{2}, I_{3}$ and $I_{4}$ are smaller than $O\left(N^{1-2 / \alpha}\right)$.

(1) By (38) there exists a constant $C>0$ (not depending on $t$ and $N$ ) such that

$$
\left|e^{-i t N / B_{N}}\left(\varphi_{X}\left(t / B_{N}\right)\right)^{N}-\varphi(t)\right| \leq|\varphi(t)|\left(e^{C t^{2} N / B_{N}^{2}}-1\right), \quad N \in \mathbb{N}, t \in \mathbb{R} .
$$

Applying the inequality $e^{x}-1 \leq x e^{x}, x \in \mathbb{R}$, to the point $x:=C t^{2} N / B_{N}^{2}$ yields

$$
\left|e^{-i t N / B_{N}}\left(\varphi_{X}\left(t / B_{N}\right)\right)^{N}-\varphi(t)\right| \leq|\varphi(t)| C t^{2} \frac{N}{B_{N}^{2}} e^{C t^{2} N / B_{N}^{2}}, \quad N \in \mathbb{N}, t \in \mathbb{R} .
$$

For all $|t| \leq k_{N}$ we have $C t^{2} N / B_{N}^{2} \leq C$ and it follows that

$$
\left|e^{-i t N / B_{N}}\left(\varphi_{X}\left(t / B_{N}\right)\right)^{N}-\varphi(t)\right| \leq C e^{C} \frac{N}{B_{N}^{2}} t^{2}|\varphi(t)|, \quad N \in \mathbb{N},|t| \leq k_{N} .
$$

Since, by $26,, \int_{-\infty}^{\infty} t^{2}|\varphi(t)| d t<\infty$, it follows for all $N \in \mathbb{N}$ that $I_{1} \leq C e^{C}\left(N / B_{N}^{2}\right) \int_{-\infty}^{\infty} t^{2}|\varphi(t)| d t=$ $O\left(N^{1-2 / \alpha}\right)$ as required.

(2) As in the proof of Theorem 4.2.1 of [12] it follows that, for any $\delta \in(0, \alpha)$, there exist constants $c(\delta) \in(0, \infty)$ and $\varepsilon(\delta)>0$ not depending on $N$ and a constant $N_{0}(\delta) \in \mathbb{N}$ such that $\left|\varphi_{X}\left(t / B_{N}\right)\right|^{N} \leq$ $e^{-c(\delta)|t|^{\delta}}$ for all $|t| \leq \varepsilon(\delta) B_{N}$ and all $N \geq N_{0}(\delta)$. Thus, choosing $\delta:=\alpha / 2$ and using the notation $c:=c(\alpha / 2)$ and $\varepsilon:=\varepsilon(\alpha / 2)$, it follows for all $N \geq N_{0}(\alpha / 2)$ that

$$
I_{2} \leq \int_{k_{N} \leq|t| \leq \varepsilon B_{N}} e^{-c|t|^{\alpha / 2}} d t \leq 2 \int_{k_{N}}^{\infty} e^{-c t^{\alpha / 2}} d t \leq 2 \int_{k_{N}}^{\infty} e^{-c \sqrt{t}} d t \sim \frac{4}{c} \sqrt{k_{N}} e^{-c \sqrt{k_{N}}},
$$

a quantity being smaller than $O\left(N^{\beta}\right)$ for any $\beta<0$, so in particular smaller than $O\left(N^{1-2 / \alpha}\right)$.

(3) From [12, Theorem 1.4.2] it follows that there exists a constant $q \in(0,1)$ such that $\left|\varphi_{X}(u)\right| \leq q$ for all $u \in[\varepsilon, \pi]$. Thus, $I_{3}=B_{N} \int_{\varepsilon \leq|u| \leq \pi}\left|\varphi_{X}(u)\right|^{N} d u \leq 2 \pi B_{N} q^{N}$, a quantity being smaller than $O\left(N^{\beta}\right)$ for any $\beta<0$.

(4) In order to estimate the integral $I_{4}$ define $c:=\theta \kappa r^{\alpha} \in(0, \infty)$ and $D:=-c \cos \frac{\pi \alpha}{2} \in(0, \infty)$ for convenience. We have

$$
I_{4}=\int_{|t|>k_{N}} e^{c \operatorname{Re}\left((-i t)^{\alpha}\right)} d t=\int_{|t|>k_{N}} e^{c|t|^{\alpha} \cos \frac{\pi \alpha}{2}} d t=2 \int_{k_{N}}^{\infty} e^{-D t^{\alpha}} d t .
$$

Noting that $k_{N} \geq 1$ for all $N \in \mathbb{N}$ and that $\alpha>1$ it follows that $I_{4} \leq 2 \int_{k_{N}}^{\infty} e^{-D t} d t=(2 / D) e^{-D k_{N}}$, again being smaller than $O\left(N^{\beta}\right)$ for any $\beta<0$.

\section{References}

[1] Berestycki, N. And Pitman, J. (2007) Gibbs distributions for random partitions generated by a fragmentation process. J. Stat. Phys. 127, 381-418. MR2314353 
[2] Billingsley, P. (1999) Convergence of Probability Measures. 2nd Edition. Wiley, New-York. MR1700749

[3] Bruisn, N. G. (1981) Asymptotic Methods in Analysis. Dover, New-York. MR0671583

[4] Charalambides, Ch. A. and Kyriakoussis, A. (1985) An asymptotic formula for the exponential polynomials and a central limit theorem for their coefficients. Discrete Math. 54, 259-270. MR0790587

[5] Comtet, L. (1974) Advanced Combinatorics. D. Reidel Publishing Company, Dordrecht, Holland. MR0460128

[6] Eldon, B. And Wakeley, J. (2006) Coalescent processes when the distribution of offspring number among individuals is highly skewed. Genetics 172, 2621-2633. MR number not available

[7] Flajolet, P. and Sedgewick, R. (2009) Analytic Combinatorics. Cambridge University Press, Cambridge. MR2483235

[8] Graham, R. L., Knuth, D. E., And Patashnik, O. (1994) Concrete Mathematics. A Foundation for Computer Science, Second Edition, Addison Wesley, Reading. MR1397498

[9] Hulllet, T. and Möhle, M. (2011) Population genetics models with skewed fertilities: a forward and backward analysis. Stochastic Models 27, 521-554. MR2827443

[10] Huillet, T. And Möhle, M. (2012) Correction on 'Population genetics models with skewed fertilities: a forward and backward analysis', Stochastic Models 28, 527-532. MR number not yet available

[11] Huillet, T. And Möhle, M. (2013+) On the extended Moran model and its relation to coalescents with multiple collisions. Theor. Popul. Biol., in press, online version already available (doi: 10.1016/j.tpb.2011.09.004)

[12] Ibragimov, I. A. And Linnik, Yu. V. (1971) Independent and Stationary Sequences of Random Variables. Wolters-Noordhoff Publishing Groningen, The Netherlands. MR0322926

[13] Janson, S. (2012) Simply generated trees, conditioned Galton-Watson trees, random allocations and condensation. Probab. Surv. 9, 103-252. MR2908619

[14] Karlin, S. And MCGregor, J. (1964) Direct product branching processes and related Markov chains. Proc. Nat. Acad. Sci. U.S.A. 51, 598-602. MR0163362

[15] Karlin, S. ANd McGregor, J. (1965) Direct product branching processes and related Markov chains. I. Calculations of rates of approach to homozygosity. Proc. Internat. Res. Sem., Springer, Berlin, pp. 111-145. MR0217892

[16] MöHle, M. (2000) Total variation distances and rates of convergence for ancestral coalescent processes in exchangeable population models. Adv. Appl. Probab. 32, 983-993. MR1808909

[17] Möhle, M. (2011) Coalescent processes derived from some compound Poisson population models. Electron. Comm. Probab. 16, 567-582. MR2846651 
[18] Möhle, M. And Sagitov, S. (2001) A classification of coalescent processes for haploid exchangeable population models. Ann. Probab. 29, 1547-1562. MR1880231

[19] Sagitov, S. (2003) Convergence to the coalescent with simultaneous multiple mergers. $J$. Appl. Probab. 40, 839-854. MR2012671. 